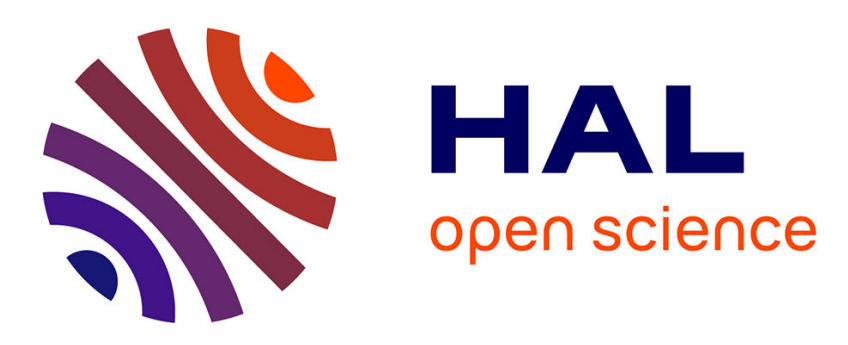

\title{
Micromechanical investigation of the influence of defects in high cycle fatigue
}

\author{
Raphaël Guerchais, Nicolas Saintier, Franck Morel, Camille Robert
}

\section{To cite this version:}

Raphaël Guerchais, Nicolas Saintier, Franck Morel, Camille Robert. Micromechanical investigation of the influence of defects in high cycle fatigue. International Journal of Fatigue, 2014, 67, pp.159-172. 10.1016/j.ijfatigue.2014.01.005 . hal-01084156

\section{HAL Id: hal-01084156 https://hal.science/hal-01084156}

Submitted on 18 Nov 2014

HAL is a multi-disciplinary open access archive for the deposit and dissemination of scientific research documents, whether they are published or not. The documents may come from teaching and research institutions in France or abroad, or from public or private research centers.
L'archive ouverte pluridisciplinaire HAL, est destinée au dépôt et à la diffusion de documents scientifiques de niveau recherche, publiés ou non, émanant des établissements d'enseignement et de recherche français ou étrangers, des laboratoires publics ou privés. 


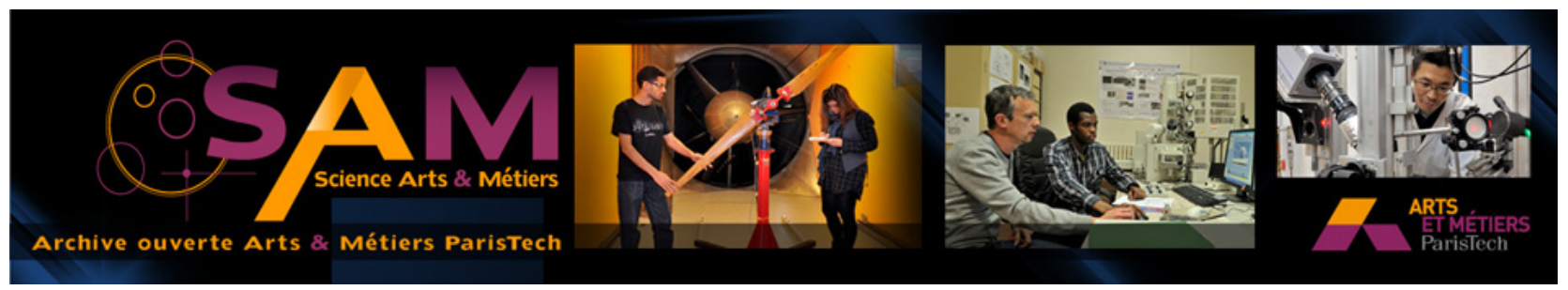

Science Arts \& Métiers (SAM)

is an open access repository that collects the work of Arts et Métiers ParisTech researchers and makes it freely available over the web where possible.

This is an author-deposited version published in: http://sam.ensam.eu

Handle ID: .http://hdl.handle.net/10985/8938

\section{To cite this version :}

Raphaël GUERCHAIS, Nicolas SAINTIER, Franck MOREL, Camille ROBERT - Micromechanical investigation of the influence of defects in high cycle fatigue - International Journal of Fatigue Vol. 67, p.159-172 - 2014 


\title{
Micromechanical investigation of the influence of defects in high cycle fatigue
}

\author{
R. Guerchais ${ }^{\mathrm{a}, \mathrm{b}, *}$, N. Saintier ${ }^{\mathrm{b}}$, F. Morel $^{\mathrm{a}}$, C. Robert $^{\mathrm{a}}$ \\ ${ }^{a}$ Arts et Metiers Paris Tech, LAMPA, 2 bd du Ronceray, 49035 Angers Cedex, France \\ ${ }^{b}$ Arts et Metiers ParisTech, I2M - UMR CNRS 5295, Université Bordeaux 1, Esplanade des Arts et \\ Métiers, 33405 Talence Cedex, France
}

\begin{abstract}
This study aims to analyse the influence of geometrical defects (notches and holes) on the high cycle fatigue behaviour of an electrolytic copper based on finite element simulations of 2D polycrystalline aggregates. In order to investigate the role of each source of anisotropy on the mechanical response at the grain scale, three different material constitutive models are assigned successively to the grains: isotropic elasticity, cubic elasticity and crystal plasticity in addition to the cubic elasticity. The significant influence of the elastic anisotropy on the mechanical response of the grains is highlighted. When considering smooth microstructures, the crystal plasticity have has a slight effect in comparison with the cubic elasticity influence. However, in the case of notched microstructures, it has been shown that the influence of the plasticity is no more negligible. Finally, the predictions of three fatigue criteria are analysed. Their ability to predict the defect size effect on the fatigue strength is evaluated thanks to a comparison with experimental data from the literature.
\end{abstract}

Keywords: High cycle fatigue, Defect, Microstructure modelling, Anisotropic elasticity, Crystal plasticity, Fatigue criterion

\section{Nomenclature}

$\gamma_{s}$ : plastic slip on the slip system $\mathrm{s}$

$\nu_{s}:$ accumulated plastic slip on the slip system $\mathrm{s}$

$\tau_{s}:$ resolved shear stress on the slip system $\mathrm{s}$

\footnotetext{
*Corresponding author. Tel: +33 (0)2 412073 27; fax: +33 (0)2 41207320
}

Email address: raphael.guerchais@ensam.eu (R. Guerchais) 


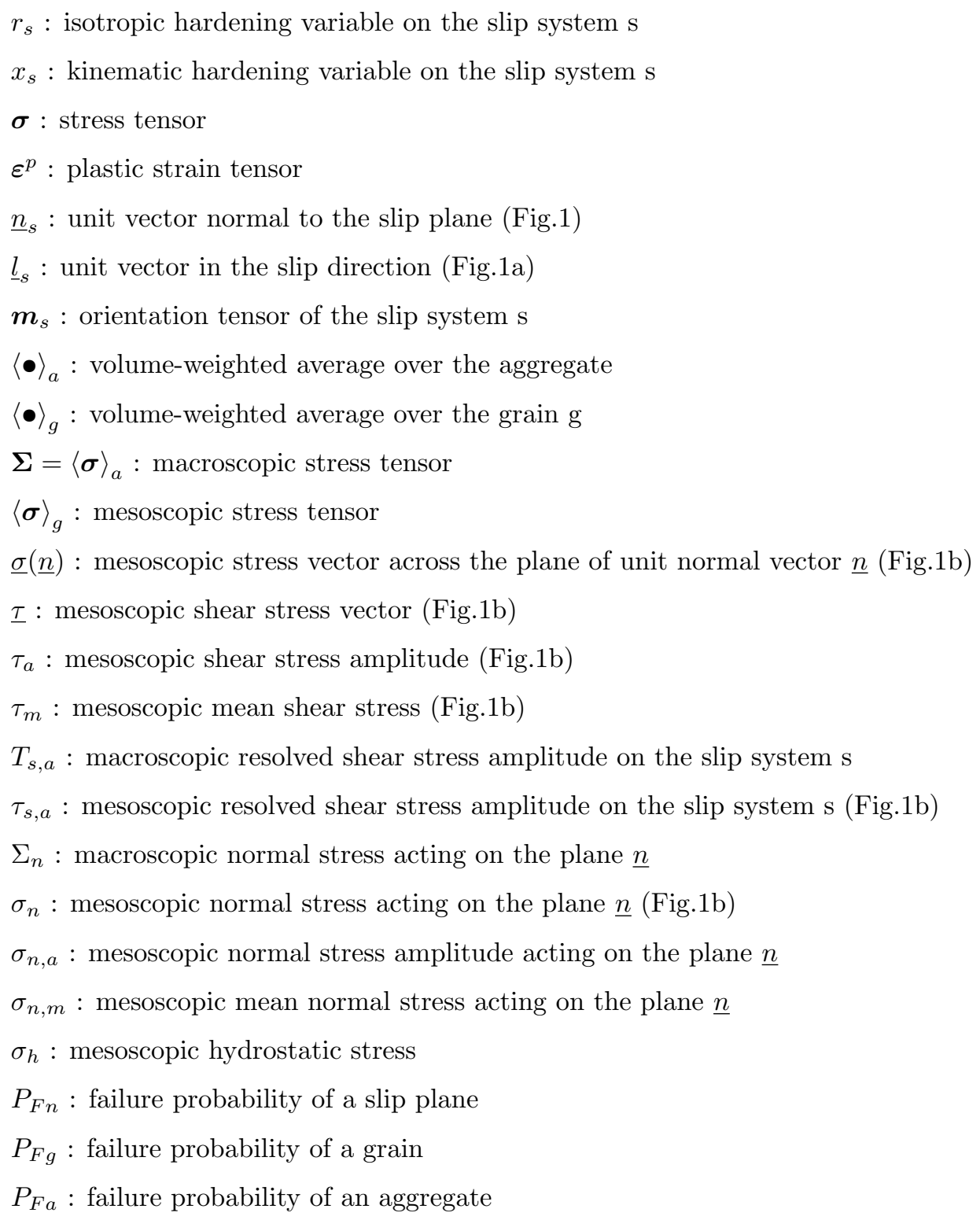

\section{Introduction}

The ability to assess the effects of small defects on the high cycle fatigue (HCF) strength appears to be crucial from the design point of view as the stress concentrations induced by these defects favour fatigue crack initiation. Several authors have established fatigue criteria 
taking into account accurately the detrimental influence of defects on the fatigue limits in tension [1], in torsion [2] and in combined tension and torsion [3]. However, although the practical interest of these approaches is undeniable, they often involve a material characteristic length whose physical meaning is unclear. Besides, some of them do not allow to account for a complex defect geometry. Moreover, these methods neglect the variabilities of the microstructure in the vicinity of the defect and thus can not reflect the scatter observed in the HCF strength of metallic materials. This scatter is often explained by the anisotropic elasto-plastic behaviour of individual grains leading to a highly heterogeneous distribution of plastic slip. Since fatigue crack initiation is a local phenomenon, intimately related to the plastic activity at the crystal scale, it seems relevant to evaluate the mesoscopic mechanical quantities (i.e. the average mechanical quantities at the grain scale) the HCF behaviour of metallic materials. Unfortunately no simple method exists to precisely estimate these quantities due to the complexity of the morphology and of the behaviour of the grains constituting a metal. Localisation schemes are a common way to relate the mechanical response of each grain to the macroscopic loading applied to a polycrystal. However, they encounter difficulties when estimating the local mechanical fields in the presence of defects whose size is comparable to the characteristic length of the microstructure (i.e. the mean grain size).

An alternative way to estimate these mechanical fields is to perform finite element (FE) analysis of explicitly modelled polycrystalline aggregates. This promising approach allows to take into account microstructural features generally neglected in the localisation schemes and to deepen the analysis of the mesoscopic mechanical responses of metals under cyclic loading. In recent years, several works have involved this kind of numerical simulations to contribute to the study of the HCF behaviour. For instance, Bennett et al. [4] have analysed the distribution of fatigue crack initiation parameters inspired from well-known HCF criteria. This study was enriched by the work of Guilhem et al. [5] in which the mechanical response of the grains is studied according to their positions in the aggregate (for instance at the free surface or in the core), their orientations and those of the neighboring grains. Some studies have highlighted the important role played by the anisotropic elasticity on the mechanical responses at the grain scale in several metallic materials with a face-centred cubic (FCC) structure $[6,7,8]$ and a body-centred cubic (BCC) structure [9]. Moreover, FE simulations of polycrystalline aggregates have recently been used to analyse the influence of defects on the 
high cycle fatigue strength. For example, the effect of a rough surface has been investigated in $[10,11]$ and the influence of the crystallographic orientations and of the defect size and acuity have been extensively studied by Owolabi et al. [12] in the case of semicircular notches. In the present work, which falls within this framework, two points are addressed:

- A numerical analysis is conducted on notched microstructures, based on push-pull fatigue limits determined by Lukàš et al. [1] thanks to notched specimens made of electrolytic copper. In a first step, the objective is to analyse the mechanical responses of the grains in microstructures cyclically loaded at a stress amplitude corresponding to the macroscopic average fatigue limit. This analysis focuses more specifically on the effects of the constitutive model used at the grain scale and the notch on the mesoscopic mechanical responses. In a second step, a comparison is carried out between the predictions provided by three different criteria with the experimental average fatigue limits.

- A qualitative numerical study is made on holed microstructure, in order to compare the influence of a defect on the mesoscopic mechanical response for different loading conditions: fully reversed tension and fully reversed shear. The fatigue limits predicted by one of the studied criterion are determined and compared to experimental trends

\section{Modelling approach}

\subsection{Constitutive material models at the grain scale}

The anisotropic behaviour of the grains is due, on the one hand, to the elastic behaviour and, on the other hand, to the crystallographic nature of the plastic slip. In FCC structure, as for pure copper, the elastic behaviour is cubic and the plastic slip occurs along the $\{111\}$ planes in the $\langle 110\rangle$ directions which correspond respectively to the closed-packed planes and directions of this crystal structure. In order to dissociate the effect of each sources of anisotropy on the mesoscopic mechanical responses, three constitutive models, assigned to the grains, are investigated:

- Linear isotropic elasticity;

- Linear cubic elasticity; 
- Linear cubic elasticity with crystal plasticity.

In each case, a Hooke's law is used to describe the elastic behaviour. In the first case, an isotropic elastic behaviour is considered and is defined by the Young's Modulus $E$ and the Poisson's ratio $\nu$. In the second and third cases, cubic elasticity is considered and completely characterized by three coefficients defined in the crystal coordinate system: $C_{1111}, C_{1122}$ and $C_{1212}$. After homogenisation, when considering an isotropic texture, the cubic elastic model is equivalent to the isotropic elastic model at the macroscopic scale.

Finally, crystal plasticity is described by a single crystal visco-plastic model proposed by Méric et al. [13]. In this constitutive model, the plastic slip rate $\dot{\gamma}_{s}$ on a slip system $s$ is governed by a Norton-type flow rule (Eq. 1) involving the resolved shear stress $\tau_{s}$ acting on $s$ and an isotropic hardening variable $r_{s}$ associated to the slip system $s$.

$$
\dot{\gamma}_{s}=\left\langle\frac{\left|\tau_{s}\right|-r_{0}-r_{s}}{K}\right\rangle_{+}^{n} \operatorname{sgn}\left(\tau_{s}\right)=\dot{\nu}_{s} \operatorname{sgn}\left(\tau_{s}\right)
$$

where $K$ and $n$ are the parameters defining the viscosity and $r_{0}$ corresponds to the critical resolved shear stress. In this equation, $\langle x\rangle_{+}=\max (x, 0)$. The resolved shear stress $\tau_{s}$ acting on $s$ is computed from the stress tensor $\boldsymbol{\sigma}$ by means of the orientation tensor $\boldsymbol{m}_{s}$ (Eqs 2 and 3). From $\boldsymbol{m}_{s}$, it is then possible to compute the plastic strain rate tensor $\dot{\boldsymbol{\varepsilon}}^{p}$ knowing the plastic slip rate $\dot{\gamma}_{s}$ occurring on each slip system $s$ (Eq. 4).

$$
\begin{gathered}
\tau_{s}=\boldsymbol{m}_{s}: \boldsymbol{\sigma} \\
\boldsymbol{m}_{s}=\frac{\left(\underline{n}_{s} \otimes \underline{l}_{s}+\underline{l}_{s} \otimes \underline{n}_{s}\right)}{2} \\
\dot{\boldsymbol{\varepsilon}}^{p}=\sum_{s} \dot{\gamma}_{s} \boldsymbol{m}_{s}
\end{gathered}
$$

The evolution law of the isotropic hardening variable $r_{s}$ is given by Eqs 5 .

$$
r_{s}=Q \sum_{r} h_{s r}\left(1-e^{-b \nu_{r}}\right)
$$

$h_{s r}$ terms represent the components of the interaction matrix, introduced by Franciosi [14] in order to account for the influence of the accumulated plastic slip $\nu_{r}$ on the slip system $r$ on the hardening of the slip system $s . Q$ and $b$ are the isotropic hardening parameters.

The material parameters values used in the FE simulation, identified by Méric et al. [13] and Gérard [15], are summarised in Table 1. 


\subsection{Finite element modelling}

\subsubsection{Notched microstructures}

In order to reproduce numerically, in a reasonable computation time, the fatigue tests conducted by Lukàš et al. [1] using finite element simulations of polycrystalline aggregates, a simplified geometry of the specimen is modelled (see Fig. 2). The first simplification is the use of 2-dimensional geometries instead of 3D semicircular notches considered in [1]. The second one consists in explicitly modelling the microstructure only in the notch area. An isotropic matrix embeds the polycrystal so that the ratio between the half-width of the specimen used in [1] and the notch radius is respected for all geometries. The use of an isotropic matrix is a common practice in studies of notched microstructures (see for instance [16] and [12]). The dimensions presented in Fig. 2 have been chosen such as the smooth polycrystalline aggregate contains 200 grains for a mean grain size of $50 \mu \mathrm{m}$.

The process used to generate the 2-dimensional polycrystalline aggregates geometries is described in [7]. Both smooth and notched microstructures are used. The notches considered in this work are semi-circular and 3 notch radii $\rho$ are studied: 40, 80 and $120 \mu m$. Finally, the finite element mesh of the CAD of the microstructure is generated using Gmsh [17]. Three-node triangular finite elements, with linear interpolation and generalised plane strain hypothesis, are used. Each grain is discretised in average with 100 elements. A finite element mesh illustration of a microstructure embedded in a matrix is shown in Fig.3. The mesh of the grains may appear coarse in comparison with the recommended discretisation suggested by Diard et al. [18]. The authors state that, for 3-dimensional microstructures, approximately 350 integration points in average per grain are necessary to correctly estimate the average values per grain of mechanical quantities which is the scale investigated in this study. However, it may seem reasonable to assume that the minimum number of integration points can be lower for $2 \mathrm{D}$ microstructures. In order to confirm this hypothesis, a comparison between the results obtained from a microstructure loaded in tension and discretised with 3-node triangular linear elements and 6-node triangular quadratic elements is undertaken. The grains contains in average 100 integration points and 300 integration points for the microstructures meshed respectively with 3-node triangular elements and with 6-node triangular elements. The mechanical quantities chosen for comparison are the mesoscopic von Mises stress and the mesoscopic hydrostatic stress which are computed from the stress tensors averaged per 
grain $\langle\boldsymbol{\sigma}\rangle_{g}$ obtained from the last increment of the FE simulations. Those two quantities are selected as they give a good view on the deviation that can be observed on the deviatoric and hydrostatic part of the mesoscopic stress tensor for the two mesh. It has been observed that for $80 \%$ of the grains, the relative differences of the mesoscopic von Mises stresses between the two meshes do not exceed $1 \%$. The maximum relative difference is lower than $4.5 \%$ for that mechanical quantity. Concerning the mesoscopic hydrostatic stress, it turns out that for $95 \%$ of the grains, the relative difference between the two meshes does not exceed $3 \%$. However, relatives differences greater than $5 \%$ have been found. This case occurs only for the grains with a low mesoscopic stress (i.e. $<30 \%$ of the maximum mesoscopic hydrostatic stress of the aggregate). In these conditions, the adopted discretisation seems acceptable.

For each defect size studied, three geometries containing approximately 200 equiaxed grains and ten orientations sets are used. Orientation sets are composed by triplet of Euler angles chosen such as to represent an isotropic texture. Each triplet of Euler angles defines the orientation of one crystal frame with respect to the reference frame of the aggregate. As a result, the response of 30 different microstructures is investigated per defect size. Computations are performed to simulate fully reversed load control fatigue tests. Such loading cases are modelled by imposing a displacement $U_{2}=0$ to the lower edge and a cyclic macroscopic stress $\Sigma_{22}(t)=\Sigma_{22, a} \sin (\pi t)$ to the upper edge of the matrix. A displacement $U_{1}=0$ is imposed on the left edge to model the specimen symmetry. When the crystal plasticity model is assigned to the grains, 10 cycles are computed so that the aggregates reach a stabilized behaviour. In the case of purely elastic models used for the grains, thanks to the linearity of these behaviours, only one cycle is applied.

The constitutive model assigned to the isotropic matrix depends on the one applied to the grains. If the grains have a purely elastic constitutive model, an isotropic elastic law is assigned to the matrix. If the crystal plasticity model is assigned to the grains, an isotropic plastic model is added to the constitutive model of the matrix. In this time-independent plasticity model, the yield surface is described by a yield function $f$ based on the von Mises expression:

$$
f=J_{2}(\boldsymbol{\Sigma}-\boldsymbol{X})-R-\Sigma_{y}
$$

Where $\boldsymbol{\Sigma}$ is the macroscopic stress tensor, $\boldsymbol{X}$ is the kinematic hardening stress tensor, $\Sigma_{y}$ is the initial size of the yield surface and $R$ is the isotropic hardening stress. Non-linear 
kinematic and isotropic hardening rules are used, respectively Armstrong-Frederick (Eq. 8) and Voce (Eq. 7) rules. The parameters of this model $\left(\Sigma_{y}, Q, b, C\right.$ and $\left.\gamma\right)$ are identified by numerical optimisation using the Levenberg-Marquardt algorithm and considering the macroscopic response of a smooth polycrystalline aggregate of 200 grains loaded in cyclic tension as the data set so that the two models are equivalent at the macroscopic scale. The material parameters obtained are summarised in Table 2.

$$
\begin{gathered}
d R=b(Q-R) d p \\
d \boldsymbol{X}=\frac{2}{3} C d \boldsymbol{E}^{p}-\gamma \boldsymbol{X} d p
\end{gathered}
$$

The numerical simulations are conducted with ZeBuLoN FE software [19] in which the constitutive models used in this study (i.e. the cubic elasticity and the single-crystal viscoplasticity) are already implemented.

\subsubsection{Holed microstructures}

The modelling of holed microstructures is similar to the one of the notched microstructures : the geometries are in 2-dimension and the polycrystalline aggregates, containing 200 grains, are embedded in an isotropic matrix. The geometry and its dimensions are shown in Fig. 4. As for notched microstructure, smooth polycrystalline aggregates are computed for comparison. The defect considered is a circular hole and 3 radii $\rho$ are studied: 28.3, 56.6 and $84.9 \mu \mathrm{m}$. The same finite elements and meshed density as in the case of notched microstructures are used. For each defect size studied, three geometries containing approximately 200 equiaxed grains and ten orientations sets are used. As a result, the response of 30 different microstructures is investigated per defect size. Two loading cases are studied: fully reversed tension and fully reversed shear. To model the fully reversed tension, a displacement $U_{2}=0$ and a cyclic macroscopic stress $\Sigma_{22}(t)=\Sigma_{22, a} \sin (\omega t)$ are imposed respectively on the lower edge and on the upper edge of the matrix. The fully reversed shear is modelled by imposing a cyclic macroscopic stress $\Sigma_{12}(t)=\Sigma_{12, a} \sin (\omega t)$ on the upper and right edges of the matrix which are oriented respectively along the $\mathrm{x}$-axis and the $\mathrm{y}$-axis. An opposite macroscopic stress $-\Sigma_{12}(t)$ is imposed on the lower and left edges of the matrix which are also oriented respectively along the $\mathrm{x}$-axis and the $\mathrm{y}$-axis. In order to limit the computation time, only elastic models are assigned to the grains and an isotropic elastic law is assigned to the matrix. 
This modelling choice seems adequate for a qualitative analysis of the influence of defect on the fatigue strength in fully reversed tension and shear. Thanks to the linearity of these behaviours, only one cycle is applied.

\section{Fatigue criteria}

The predictions of three different fatigue criteria are studied in this work. Their expressions derive from multiaxial HCF fatigue criteria based on the mesoscopic approaches proposed by Dang Van [20], Papadopoulos [21] and Morel and Huyen [22]. The main change made on these criteria is the replacement of the macroscopic mechanical quantities by mesoscopic mechanical quantities, i.e. the quantities computed from the stress tensors averaged per grain $\langle\boldsymbol{\sigma}\rangle_{g}$ which are obtained from the last loading cycle of the FE simulations.

\subsection{Critical plane-based criterion}

The criterion proposed by Dang Van is based on the assumption that the fatigue crack initiation in one grain leads to the failure of the entire polycrystalline aggregate. To keep this idea, a criterion checking that no crack initiates during the last loading cycle in each slip plane contained in the polycrystal is proposed (Eq. 9). With this relation, which is similar to the one proposed by Dang Van, it is assumed that the fatigue failure is prevented as long as the inequality is satisfied.

$$
\sigma_{D V}=\max _{\underline{n}}\left[\max _{t}\left[\left\|\underline{\tau}(\underline{n}, t)-\underline{\tau}_{m}(\underline{n})\right\|+\alpha_{D V} \sigma_{h}(t)\right]\right] \leq \beta_{D V}
$$

$\alpha_{D V}$ and $\beta_{D V}$ are two material parameters. $\underline{\tau}(\underline{n}, t)$ represents the mesoscopic shear stress vector acting on the slip plane $\underline{n}, \underline{\tau}_{m}(\underline{n})$ the mesoscopic mean shear stress vector acting on the slip plane $\underline{n}$ and $\sigma_{h}(t)$ the mesoscopic hydrostatic pressure. As the direction of the mesoscopic shear stress vector $\underline{\tau}(\underline{n}, t)$ generally changes with time, the end of this vector described a path $\Gamma$ (Fig.1b) which is, in our case, computed during the last loading cycle. Under these conditions, an appropriate definition of the mesoscopic mean shear stress vector $\underline{\tau}_{m}(\underline{n})$, to respect its uniqueness [23], consists to define $\underline{\tau}_{m}(\underline{n})$ as the vector $\underline{O \Omega}$ with $O$ being the origin of the shear stress vector and $\Omega$ being the centre of the smallest circle circumscribing the path $\Gamma$ (Fig.1b). The randomised algorithm summarised in [23] is used to efficiently find the minimum enclosing circle of the path of each slip plane. 


\subsection{Integral criterion}

Like Dang Van, Papadopoulos has developed a fatigue criterion at the grain scale based on the concept of elastic shakedown. In this mesoscopic fatigue criterion, a fatigue crack does not initiate in a grain if the accumulated plastic slip on a given slip system does not exceed a threshold.

Papadopoulos wisely noticed that the engineering fatigue limit is not considered as the stress amplitude at which there is no crack initiation. The author relies on the fact that non-propagating small fatigue cracks can be observed in specimens loaded below their conventional fatigue limit. This leads Papadopoulos to propose a criterion, given in Eq.10, based on an estimate of the average accumulated plastic slip of all the slip systems contained in a representative volume element (RVE). This criterion is expressed as a function of the quadratic mean, along every slip systems in the polycrystal, of the macroscopic resolved shear stress amplitude $T_{s, a}$ (Eq. 11) and the average, along every slip planes in the polycrystal, of the macroscopic normal stess $\Sigma_{n}$ (Eq. 12).

$$
\begin{gathered}
\sqrt{\left\langle T_{s, a}^{2}\right\rangle}+\alpha \max _{t}\left[\left\langle\Sigma_{n}(t)\right\rangle\right] \leq \beta \\
\sqrt{\left\langle T_{s, a}^{2}\right\rangle}=\sqrt{5} \sqrt{\frac{1}{8 \pi^{2}} \int_{\varphi=0}^{2 \pi} \int_{\theta=0}^{\pi} \int_{\chi=0}^{2 \pi} T_{s, a}^{2} d \chi \sin (\theta) d \theta d \varphi} \\
\left\langle\Sigma_{n}(t)\right\rangle=\frac{1}{8 \pi^{2}} \int_{\varphi=0}^{2 \pi} \int_{\theta=0}^{\pi} \int_{\chi=0}^{2 \pi} \Sigma_{n}(t) d \chi \sin (\theta) d \theta d \varphi
\end{gathered}
$$

In this work, the form of this criterion is preserved (Eq. 13) but mesoscopic quantities are used instead of the macroscopic ones $\left(\tau_{s, a}\right.$ and $\sigma_{n}$ replace respectively $T_{s, a}$ and $\left.\Sigma_{n}\right)$ :

$$
\sigma_{P}=\sqrt{\left\langle\tau_{s, a}^{2}\right\rangle}+\alpha_{P} \max _{t}\left[\left\langle\sigma_{n}(t)\right\rangle\right] \leq \beta_{P}
$$

where $\alpha_{P}$ and $\beta_{P}$ correspond to the material parameters. As the microstructures studied

contain a finite number of grains and slip systems, $\sqrt{\left\langle\tau_{s, a}^{2}\right\rangle}$ and $\left\langle\sigma_{n}\right\rangle$ become

$$
\begin{gathered}
\sqrt{\left\langle\tau_{s, a}^{2}\right\rangle}=\sqrt{5} \sqrt{\frac{1}{N_{s}} \sum_{g=1}^{N_{g}}\left[f(g) \sum_{s=1}^{N_{s}} \tau_{s, a}^{2}(g, s)\right]} \\
\left\langle\sigma_{n}(t)\right\rangle=\frac{1}{N_{p}} \sum_{g=1}^{N_{g}}\left[f(g) \sum_{p=1}^{N_{p}} \sigma_{n}(g, p, t)\right]
\end{gathered}
$$


where $N_{s}$ and $N_{p}$ are respectively the number of slip systems in a grain and the number of slip planes in a grain. In Eqs 14 and 15, $f(g)$ represents the volume fraction of the grain $g$. For the sake of simplicity, as the grains in the microstructure have approximately the same volume, $f(g)$ is assumed to be equal to $1 / N_{g}$ with $N_{g}$ being the number of grains in the considered microstructure.

\subsection{Probabilistic fatigue criterion}

Morel and Huyen have proposed a criterion based on the assumption that the fatigue crack initiation threshold at the grain scale follows a Weibull distribution which led them to define a failure probability for each grain contained in a polycrystalline aggregate [22]. In order to estimate the failure probability of the polycrystal, the authors then applied the weakest-link hypothesis. This reasoning is repeated below.

First, a fatigue crack is assumed to initiate in a slip plane of normal $\underline{n}$ if the amplitude of shear stress $\tau_{a}$ acting on this plane exceeds a threshold $\tau_{a}^{t h} . \tau_{a}$ is defined as the radius of the smallest circle circumscribing the path described by $\underline{\tau}(\underline{n}, t)$ during the last loading cycle. Once again, the randomised algorithm [23] is used to determine this circle. The threshold $\tau_{a}^{t h}$ is then supposed to be a random variable following a Weibull distribution characterised by a shape parameter $m$ and a scale parameter $\tau_{0}$. Thus, the failure probability of the slip plane can be expressed by:

$$
P_{F n}=P\left(\tau_{a} \geq \tau_{a}^{t h}\right)=1-\exp \left[-\left(\frac{\tau_{a}}{\tau_{0}}\right)^{m}\right]
$$

The normal stress effect on the fatigue strength is taken into account by considering that $\tau_{0}$ depends on the normal stress amplitude $\sigma_{n, a}$ and on the mean normal stress $\sigma_{n, m}$ acting on the slip plane of normal $\underline{n}$ (Eq. 17).

$$
\tau_{0}=\tau_{0}^{\prime} \frac{1-\gamma \sigma_{n, m}}{1+\alpha\left(\sigma_{n, a} / \tau_{a}\right)}
$$

In Eq. 17, $\tau_{0}^{\prime}, \gamma$ and $\alpha$ are material parameters.

The failure probability $P_{F g}$ of a grain $g$ is supposed to correspond to the maximum failure among the failure probabilities of its slip planes (Eq. 18). This assumption constitutes the major difference with respect to the initial criterion. Indeed, the weakest-link hypothesis was used by Morel and Huyen to determined the failure probability at the grain scale $P_{F g}$.

$$
P_{F g}=\max _{\underline{n} \in g}\left[P_{F n}\right]
$$


Finally, the weakest-link hypothesis is applied to determine the failure probability of a polycrystalline aggregate $P_{F a}$ which leads to the following expression:

$$
1-P_{F a}=\prod_{g=1}^{N_{g}}\left(1-P_{F g}\right)
$$

where $N_{g}$ is the number of grain constituting the polycrystalline aggregate. The use of the weakest-link hypothesis is justified by the fact that in $\mathrm{HCF}$ regime, the failure is driven by the initiation and the propagation of a single crack more than the initiation and the coalescence of a large number of cracks.

\subsection{Identification of the fatigue criteria parameters and predictions of the fatigue limits}

For each constitutive models assigned to the grains, the parameters of the criteria are identified thanks to numerical simulations' results on smooth microstructures loaded, at the average fatigue limit level, in fully reversed tension and in fully reversed shear. Moreover, as the probabilistic criterion has a parameter which defines the sensitivity to the mean normal stress, the results obtained from a third loading case are needed. A simple loading case inducing mesoscopic mean normal stresses is chosen: cyclic tension with a stress ratio $R=0$.

The average fatigue limits in fully reversed tension and in fully reversed torsion, for an electrolytic copper, have been determined respectively by Lukàš [1] and Ravilly (reported in [24]) and are $s_{-1}=73 \mathrm{MPa}$ and $t_{-1}=44 \mathrm{MPa}$. The fatigue limit in tension with $R_{\sigma}=0$ has been estimated with the Gerber relation which gives $s_{0}=66.4 \mathrm{MPa}$ for a $U T S=220$ $M P a$.

For the integral and the critical plane-based criteria, the parameters are identified such that $\frac{\sigma_{D V}}{\beta_{D V}}$ and $\frac{\sigma_{P}}{\beta_{P}}$ are, in average on the 30 realisations, equal to 1 in fully reversed tension and in fully reversed shear. Regarding the probabilistic criterion, the procedure is similar excepted that the shape parameter $m$ is imposed. Two values are chosen: 5 and 20 . The other parameters are identified such that $P_{F a}$ is, in average, equal to $50 \%$ for each of the three loading cases.

Once the parameters identified, the fatigue criteria are used to predict the fatigue limits of the notched and holed microstructures. For a given loading case, defect geometry and radius, the determination of the predicted average fatigue limit consists in searching the macroscopic stress amplitude, $\Sigma_{22, a}$ in the case of fully reversed tension and $\Sigma_{12, a}$ in the case 
of fully reversed shear, which have to be applied to the polycrystalline aggregates such as in average on the 9 realisations:

- $\sigma_{D V} / \beta_{D V}=1$ for the critical plane criterion;

- $\sigma_{P} / \beta_{P}=1$ for the integral criterion;

- $P_{F a}=50 \%$ for the probabilistic criterion.

When elastic constitutive models are used, only one FE computation per loading condition is needed to determine this loading amplitude thanks to the linearity of the mechanical response. On the contrary, in cases where crystal plasticity is assigned to the grains, the search of the fatigue limit level is an iterative process requiring several FE simulations per loading condition which leads to important computation times. For that reason, the fatigue limits are predicted only for the notched microstructures when crystal plasticity is used.

\section{Results and discussion}

The mechanical response of the polycrystalline aggregates is studied at the grain scale, for the three material constitutive models assigned to the grains, through the mesoscopic mechanical quantities, i.e. the quantities computed from the stress tensors averaged per grain $\langle\boldsymbol{\sigma}\rangle_{g}$ which are obtained from the last loading cycle of the FE simulations.

\subsection{Notched microstructures}

Before discussing the mesoscopic mechanical quantities, it would be interesting to review some features of the local mechanical fields computed from the simulations of polycrystalline aggregates using crystal plasticity. This brief analysis relies on Fig. 5 which represents the accumulated equivalent plastic slip field determined at the gauss points at the end of the $10^{\text {th }}$ loading cycle for some configurations. The same microstructure geometry is used in every case excepted that the notch radius $\rho$ increases gradually from the $1^{\text {st }}$ to the $4^{\text {th }}$ column. Moreover, the results in a given row are obtained with the same orientation set. For each defect size, the polycrystalline aggregates are loaded in fully reversed tension at the experimental average fatigue limit level excepted in the case where $\rho=40 \mu \mathrm{m}$. For this defect size, the experimental average fatigue limit being unknown, the polycrystalline aggregates 
are loaded at same loading amplitude as the smooth microstructures. This approximation seems reasonable given that the critical defect size, below which the defect does not affect the fatigue strength, is more likely greater than $40 \mu \mathrm{m}$ (see [1] for further details).

This figure highlights the heterogeneity, both intragranular and intergranular, of plastic deformations in a polycrystallin material. A similar observation was noted by Guilhem et al. [5]. However, it is important to note that the degree of heterogeneity of the intragranular plastic slip is widely underestimated in these FE simulations for two reasons:

- the mesh is not sufficiently refined to correctly determine the local extrema either in terms of stress or strain (see [18] for further informations).

- the modelling does not take into account the formation of dislocation structures in the grains; thus, the plastic slip localisation in slip bands is neglected.

Looking now at the case of smooth microstructures, it can observed that the crystalline orientations have a significant influence on the position and the magnitude of the plastic slip. Moreover, it is worth noting that in the case of notched microstructures with a notch radius $\rho=40 \mu \mathrm{m}$, the accumulated equivalent plastic slip reached at the notch root have the same order of magnitude as the one obtained in the most plastified region of the rest of the microstructure. The conclusions are different for larger notches $(\rho=80 \mu \mathrm{m}$ and $\rho=120$ $\mu m$ ). Indeed, as one might expect, the plastic slip localisation occurs in the vicinity of the notch while the rest of the microstructure is subjected to a low plastic slip activity. However, the effect of the crystallographic orientations on the intensity and the position at the notch root of the plastic slip localisation is still noticeable.

\subsubsection{Effect of the constitutive models on the mesoscopic mechanical responses}

Distributions of the shear and normal stress amplitudes in the smooth and notched polycrystalline aggregates. The mechanical responses of the grains in polycrystalline aggregates have been studied through several mechanical quantities: components of the stress and strain tensors expressed in the reference frame of the aggregate [5, 7, 25, 11], von Mises equivalent stress $[10,11]$, mesoscopic shear and hydrostatic stresses $[16,7]$, fatigue criterion parameters $[4,5,12,26,11]$, etc. To complete these analyses, the distributions of two mechanical quantities relevant in fatigue are investigated: the mesoscopic shear stress amplitude $\tau_{a}$ and 
the mesoscopic normal stress amplitude $\sigma_{n, a}$ which are both used in the probabilistic fatigue criterion. The mesoscopic mechanical response during the last loading cycle of the smooth and notched microstructures is studied for the three constitutive models. For each defect size, the polycrystalline aggregates are loaded in fully reversed tension at the experimental average fatigue limit level.

For each constitutive model and each defect size, the mechanical response of the slip planes, obtained with 30 microstructures (three polycrystalline aggregates and ten orientation sets), is reported in a diagram $\tau_{a}-\sigma_{n, a}$ in Fig. 6. On this figure, the results obtained with the isotropic elasticity, the cubic elasticity and the crystal plasticity with the cubic elasticity are presented respectively in the $1^{\text {st }}, 2^{\text {nd }}$ and $3^{\text {rd }}$ column. The diagrams in the $1^{\text {st }}$ row concern the results obtained on the smooth microstructures while the $2^{\text {nd }}, 3^{\text {rd }}$ and $4^{\text {th }}$ rows present the results from the FE computations on the notched microstructures (respectively $\rho=40$ $\mu m, \rho=80 \mu m$ and $\rho=120 \mu m)$. The dashed curves represent a partial estimate of the convex envelopes of the sets of points. Moreover, the distribution, the mean value $\mu$ and the maximum value of both $\tau_{a}$ and $\sigma_{n, a}$ are also given for each diagram.

The results presented in Fig.6 clearly show that the distributions of the considered mechanical quantities are significantly affected when the isotropic elastic model is replaced by the cubic elastic model. Indeed, taking into account the anisotropic elastic behaviour of the grains leads to a notable increase in mean and maximum values of the normal stress amplitude, especially in the case of smooth microstructures. The effect of the cubic elasticity on the distribution of the shear stress amplitude is different: the mean values slightly decrease for every defect sizes and the maximum values increase excepted for the largest notch radius. Nevertheless, the increase in the maximum values of $\tau_{a}$ is only significant in the case of defect-free microstructures. Actually, the most noticeable change occurs on the skewness, making the distributions of $\tau_{a}$ more symmetric when the cubic elastic model is assigned to the grains.

The addition of the crystal plasticity affects only slightly the distributions of the mechanical quantities in the case of smooth microstructures (Fig. 6a): the distribution of $\sigma_{n, a}$ is almost unchanged and the maximum values of $\tau_{a}$ are reduced. The small effect of the crystal plasticity compared to the one of the cubic elasticity is due to the strong anisotropic elastic behaviour of the copper and to the low loading amplitudes in high cycle fatigue leading to a 
moderate plastic flow. In the case of notched microstructures (Fig. 6b, c, d), the influence of the crystal plasticity on the mechanical response is more pronounced. Indeed, although the mean values of $\tau_{a}$ are not changed, the maximum values noticeably decrease. This reduction is due to the fact that the hardening limits the stress level reached in the grains undergoing high stress in the vicinity of the notch.

Distributions of the mean normal stresses in the smooth and notched polycrystalline aggregates. One last mechanical quantity used in the probabilistic fatigue criterion has not yet been discussed: the mesoscopic mean normal stress $\sigma_{n, m}$. This variable may seem superfluous as only fully reversed tension is considered in the present study. Indeed, for this loading case, the mesoscopic mean normal stresses are necessarily equal to zero when a purely elastic constitutive model is assigned to the grains. However, this observation is no longer true when the crystal plasticity is used in the constitutive model of the grains. Indeed, the plastic strain incompatibilities between the grains induce a residual stress field leading to non-zero mesoscopic mean normal stresses. In these conditions, it may be interesting to have a sense of the magnitude of mesoscopic mean normal stresses with respect to that of mesoscopic normal stress amplitudes. Thus, in order to analyse, for each defect size, the effect of the crystal plasticity on the distribution of the mesoscopic mean normal stress and to compare this distribution to that of the mesoscopic normal stress amplitude, the mechanical responses of each slip planes are presented in $\sigma_{n, m^{-}} \sigma_{n, a}$ diagrams in figure 7. The distribution, the mean value $\mu$ and the maximum value of both $\sigma_{n, m}$ and $\sigma_{n, a}$ are given for each diagram.

From figure 7 , it can be noticed that although the maximum values of the mesoscopic mean normal stresses are lower than those reached by the mesoscopic normal stress amplitudes, these mean normal stresses are not negligible. Indeed, in some highly stressed slip planes, the mesoscopic mean normal stress $\sigma_{n, m}$ is only twice lower than the mesoscopic normal stress amplitude $\sigma_{n, a}$.

Critical slip planes orientations. The orientations of the most critical slip planes in fully reversed tension are qualitatively studied, for each defect size and each constitutive model, thanks to the probabilistic fatigue criterion. This analysis is conducted by computing the failure probability $P_{F g}$ of each grain in ten FE simulations (one polycrystalline aggregate and ten orientation sets). The failure probability $P_{F g}$ of a given grain is then associated to 
the unit vector $\underline{n}_{s}$ normal to the corresponding slip plane. Each unit normal vector $\underline{n}_{s}$ is represented by a point in one of the pole figures gathered in Fig.8, thanks to a stereographic projection, in the plane of normal vector $\underline{e}_{3}$ (the reference frame is illustrated in Fig.3). The grey level associated to the points corresponds to the magnitude of the failure probability $P_{F g}$. On this figure, the results obtained with the isotropic elasticity, the cubic elasticity and the crystal plasticity with the cubic elasticity are presented respectively in the $1^{\text {st }}, 2^{\text {nd }}$ and $3^{\text {rd }}$ column. The pole figures in the $1^{\text {st }}, 2^{\text {nd }}, 3^{\text {rd }}$ and $4^{\text {th }}$ rows present the results obtained respectively for the notch radii $\rho: 0 \mu m, 40 \mu m, 80 \mu m$ and $120 \mu m$. For each constitutive model, the polycrystalline aggregates are loaded at the average fatigue limit level and the tensile loading is applied in the direction of $\underline{e}_{2}$. Only the grains having a failure probability $P_{F g}>10^{-5}$ are reported.

Looking now at the pole figures obtained with the defect-free microstructures in Fig. 8a, it appears that the use of an anisotropic constitutive model (elastic or plastic) increases the scatter of the failure probabilities of the grains. Moreover, the orientations of the normal vectors of the most critical planes tend to be closer to the loading axis $\underline{e}_{2}$ when the crystal plasticity is assigned to the grains. Indeed, for this configuration, the angles between the loading axis and the normal vectors of the most critical planes are ranging approximately from $0^{\circ}$ to $45^{\circ}$ whereas the interval is about $\left[20^{\circ}, 70^{\circ}\right]$ when only purely elastic model is used. This conclusion is valid even for notched microstructures (see Fig. 8b, 8c and 8d). Furthermore, it can be noted that in presence of notch, the differences between the lowest and the highest values of the failure probabilities of the grains are notably accentuated whatever the constitutive model assigned to the grains. This finding was predictable given that only the slip planes in the vicinity of the notch undergo high stress.

\subsubsection{Predictions of the fatigue criteria}

The predictions obtained for each criterion and each constitutive models assigned to the grains are presented in Fig. 9. When the crystal plasticity is used, the predicted fatigue limits are iteratively searched and thus a significant computation time is required. For this reason, the predictions of the probabilistic fatigue criterion are determined, in the case of crystal plasticity, only for the most promising - in terms of prediction accuracy with the cubic elastic model - identified parameters set. Given the results in Fig. 9b, the parameters 
set selected is the one for which $m=20$.

Whatever the constitutive model applied at the grain scale, it can be observed in Fig. 9 that the critical plane-based criterion provides the most conservatives results whereas the predictions of the integral criterion overestimate the fatigue limits of the notched microstructures. However, it is worth noting that the fatigue limits predicted by the critical plane criterion are closer to the experimental data when the elastic and plastic anisotropy is taken into account in the FE models. On the contrary, the predictions provided by the integral criterion are almost independent of the constitutive model assigned to the grains. It has to be noted that the fatigue limits predicted by this last criterion highly depend of the number of grains considered in the vicinity of the notch. Indeed, the grains farthest from the notch, which are less stressed, overshadow the notch effect on the predicted fatigue limits. An appropriate way to improve the prediction of this criterion would be to consider in the computation of $\sigma_{P}$ only the grains or the slip planes for which the level of a "fatigue indicator parameter" is above a threshold level. This method of selection of grains to properly define a damage process zone was proposed by Owolabi et al. in [12]. In the case of the integral criterion, the fatigue indicator parameter could be, for instance, the amplitude of the shear stress acting on a slip plane $\tau_{a}$. However such an approach was not investigated in the present paper.

Furthermore, it has to be noted that the probabilistic fatigue criterion, for $m=20$, provides good estimations of the experimental fatigue limits especially in the case of the $\mathrm{FE}$ models using crystal plasticity. This criterion can be seen as a good compromise between the local approach of the critical plane-based criterion and the global approach of the integral criterion. Indeed, all the grains in the aggregate are considered to predict the failure of the polycrystal but the contribution of each grain to the fatigue failure is driven by the parameters of the fatigue crack initiation threshold distribution. The lower the standard deviation of the distribution (high $m$ value and low $\tau_{0}$ value), the higher the contribution to the failure of the most stressed grains, the less stressed grains having a negligible effect.

\subsection{Holed microstructures}

As previously, the objective is to apply the loading amplitude corresponding to the average macroscopic fatigue limit to study the mesoscopic mechanical response. However, 
unlike the case of the notched microstructures, the macroscopic fatigue limits of the holed microstructures are unknown. As the probabilistic criterion provides the most convincing predictions in the case of notched microstructures, it is used to estimate these fatigue limits both in fully reversed tension and in fully reversed shear. The shape parameter $m$ is taken equal to 20 .

\subsubsection{Effect of the constitutive models on the mesoscopic mechanical responses}

Effect of the constitutive models on the mechanical response of the smooth and holed polycrystalline aggregates. The approach followed to study the effect of the constitutive models on the mechanical responses of the grains is identical to the one described in section 4.1.1. The mesoscopic mechanical responses $\tau_{a}-\sigma_{n, a}$ of each slip planes deduced from the FE simulations of holed microstructures loaded in fully reversed tension and in fully reversed shear are respectively presented in Figs. 10 and 11.

The conclusions about the effect of the anisotropic elasticity are similar to those drawn in the case of notched microstructures. Indeed, when the cubic elastic model is assigned to the grains, the maximum and the mean values of the mesoscopic normal stress amplitudes $\sigma_{n, a}$ are notably increased compared to the isotropic elastic case and the skewness of the distributions of the mesoscopic shear stress amplitude $\tau_{a}$ is closer to 0. Furthermore, a major effect of the hole on the maximum values reached by $\tau_{a}$ and $\sigma_{n, a}$ is again observed.

Critical slip planes orientations. The analysis of the orientation of the most critical slip plane is conducted thanks to the probabilistic fatigue criterion as the same manner as in in section 4.1.1. The pole figures obtained from the FE simulations of the holed microstructures (with $\rho=0$ and $84.9 \mu \mathrm{m}$ ) loaded in fully reversed tension and in fully reversed shear are respectively gathered in Figs. 12 and 13.

The orientations of the normal vector of the critical planes predicted in fully reversed tension are similar in the notched and the holed microstructures: the normal vectors of the most critical planes are ranging approximately from $20^{\circ}$ to $70^{\circ}$ with respect to the loading axis $\underline{e}_{2}$ when only elastic model is used. Besides, the anisotropic elastic behavior induced an increase in the scatter of the failure probabilities of the grains. This conclusion is also valid in the case of the fully reversed shear (see Fig. 13). Moreover, the differences between the maximum and the minimum failure probabilities are more important in the 
holed microstructures than in the smooth microstructures whatever the loading condition considered. Furthermore, in the case of defect-free microstructures loaded in fully reversed shear, the normal vectors of the most critical planes are ranging approximately from $0^{\circ}$ to $40^{\circ}$ around $\underline{e}_{1}$ and $\underline{e}_{2}$. The orientations of these normal vectors are far more scattered in the case of holed microstructures as it can be observed in Fig. 13b.

\subsubsection{Predictions of the probabilistic fatigue criterion}

Before discussing the predictions of the probabilistic fatigue criterion, it is appropriate to review the experimental trends observed on fatigue strength of metallic material in presence of an artificial defect and under fully reversed tension and torsion. In [2], Susmel, relying on data from fatigue tests performed by Endo et Murakami [27] on specimens made of $0.46 \% \mathrm{C}$ steel with a drilled hole, stated that:

- the critical radius of the hole, below which this defect does not reduce the fatigue strength, is much larger in fully reversed torsion than in rotating bending.

- the gradient of the sloping part of the threshold in a Kitagawa-Takahashi diagram $\left(\log \left(\Sigma_{a}\right)\right.$ versus $\left.\log (\rho)\right)$ is equal to about $-1 / 6$ both for rotating bending and fully reversed torsion.

These conclusions are not verified by the results of the fatigue tests conducted by Billaudeau et al. [28] on specimen made of $0.36 \% \mathrm{C}$ steel with hemispherical defect. Indeed, in this case, the gradient of the sloping part is different from $-1 / 6$ both in fully reversed tension and in fully reversed torsion. Moreover, the fatigue strength decrease is more pronounced in tension than in torsion whatever the defect size considered. However, a common feature is observed in these studies: the increase of the defect size $\rho$ leads to an increase of the ratio $\varphi$ between the fatigue limits in torsion $t_{-1}(\rho)$ and in tension $s_{-1}(\rho)$ (Eq. 20).

$$
\varphi=\frac{t_{-1}(\rho)}{s_{-1}(\rho)}
$$

For instance, in [28] $\varphi$ reaches almost 1 in presence of an artificial defect while this ratio is initially equal to 0.72 with a smooth specimen. This trend is confirmed by the experimental fatigue limits determined by Endo [29] on specimens made of $0.37 \% \mathrm{C}$ steel with a drilled hole. In this case, $\varphi$ increases from 0.62 to 0.80 for a hole diameter ranging from 0 to 500 $\mu m$. 
The fatigue limits estimated thanks to the probabilistic fatigue criterion by taking $m=20$ are presented in Fig. 14. It is worth noting that these predictions are similar to the trends observed in [28]: the critical defect sizes in fully reversed tension and shear is not clearly identifiable and the decrease of the fatigue limit is more important in tension than in shear for each defect size studied. Moreover, as in the case of notched microstructures, the use of cubic elasticity leads only to slight changes in the predictions of the criterion with respect to those obtained with the isotropic elasticity. Besides, these predicted fatigue limits are generally more optimistic in the case of the anisotropic elastic model than in the one of the isotropic elastic model.

The analysis will now focus on the evolution of the ratio $\varphi$, predicted by the probabilistic fatigue criterion, with respect to the hole radius. These ratios, resulting from the predictions, are reported in Fig. 15. It can be noticed that the predicted ratios $\varphi$ are almost identical whatever the elastic model assigned to the grains. Furthermore, these results show that an increase in the notch radius induced an increase in the predicted ratio $\varphi$, thus demonstrating a good agreement between the predictions of the probabilistic fatigue criterion and the experimental trends.

\section{Conclusion}

The role of each source of anisotropy (elastic and plastic) on the mechanical response at the grain scale and more specifically on the distributions of shear and normal stresses have been discussed in polycrystalline aggregates with and without defect. It has been highlighted that the cubic elasticity, unlike the crystal plasticity, significantly affects these distributions of $\tau_{a}$ and $\sigma_{n, a}$ in the case of smooth microstructures. These results are attributable to the strong anisotropic elastic behaviour of copper single crystals and to the low loading amplitudes specific to high cycle fatigue leading to a moderate plastic slip activity. However, the crystal plasticity plays a more important role in the mesoscopic mechanical response when notched microstructures are considered. Indeed, the maximum values of the mesoscopic shear stress amplitude reached in the grains in the vicinity of the notch are notably reduced thanks

to the plasticity. As fatigue crack initiation occurs in the most severely stressed grains, it seems relevant to account for the crystal plasticity in the modelling to satisfactorily estimate the mechanical response of these grains. 
The predictions obtained from three different criteria using the results of the FE simulations of notched microstructures have then been studied. The approach of each criterion is different which makes them more or less sensitive to the change of constitutive model and to the defect. The predictions provided by the critical plane criterion are too conservatives as only the most critical slip plane in the polycrystal is considered to estimate the fatigue failure. The integral criterion overestimates the fatigue strength. The effect of the defect on the macroscopic fatigue limit is almost neglected by this criterion due to the fact that the mechanical response of every slip systems in the aggregate is considered in the computation of the averaged mechanical quantities used in the criterion. Thus, the grains farthest from the notch, which undergo low stress, overshadow the notch effect on the predicted fatigue limits. The probabilistic fatigue criterion is a good alternative to these two criteria. Indeed, in this criterion, every grains are considered to predict the fatigue limits but, thanks to the distribution of the fatigue crack initiation threshold, only the most critical grains contribute effectively to the fatigue failure. It follows that this criterion is able of predict satisfactorily the influence of a defect on the fatigue strength as shown in the comparison of the predicted fatigue limits with experimental data.

In the case of holed microstructures, two loading conditions have been considered: fully reversed tension and fully reversed shear. Due to the lack of experimental macroscopic fatigue limits, only a comparison between the predictions of the probabilistic fatigue criterion and the experimental trends observed in metallic materials is conducted. From the fatigue limits predicted in fully reversed tension and in fully reversed shear, it has appeared that the detrimental effect of the hole on fatigue strength is more pronounced in shear than in tension for a given defect size. Thus, an increase in the defect size $\rho$ leads to an increase in the ratio between the predicted fatigue limits in torsion and in tension which is in agreement, to the authors knowledge, with all experimental studies.

[1] P. Lukàš, L. Kunz, B. Weiss, R. Stickler, Notch size effect in fatigue, Fatigue and Fracture of Engineering Materials and Structures 12 (3) (1989) 175-186.

[2] L. Susmel, D. Taylor, A simplified approach to apply the theory of critical distances to notched components under torsional fatigue loading, International Journal of Fatigue 28 (4) (2006) 417-430. 
[3] M. Endo, I. Ishimoto, The fatigue strength of steels containing small holes under outof-phase combined loading, International Journal of Fatigue 28 (56) (2006) 592-597.

[4] V. Bennett, D. McDowell, Polycrystal orientation distribution effects on microslip in high cycle fatigue, International Journal of Fatigue 25 (1) (2003) 27-39.

[5] Y. Guilhem, S. Basseville, F. Curtit, J.-M. Stéphan, G. Cailletaud, Investigation of the effect of grain clusters on fatigue crack initiation in polycrystals, International Journal of Fatigue 32 (11) (2010) 1748-1763.

[6] M. Sauzay, Cubic elasticity and stress distribution at the free surface of polycrystals, Acta Materialia 55 (4) (2007) 1193-1202.

[7] C. Robert, N. Saintier, T. Palin-Luc, F. Morel, Micro-mechanical modelling of high cycle fatigue behaviour of metals under multiaxial loads, Mechanics of Materials 55 (2012) $112-129$.

[8] R. Guerchais, C. Robert, F. Morel, N. Saintier, Micromechanical study of the loading path effect in high cycle fatigue, International Journal of Fatigue 59 (2014) 64-75.

[9] C. A. Sweeney, W. Vorster, S. B. Leen, E. Sakurada, P. E. McHugh, F. P. E. Dunne, The role of elastic anisotropy, length scale and crystallographic slip in fatigue crack nucleation, Journal of the Mechanics and Physics of Solids 61 (5) (2013) 1224-1240.

[10] X. Fang, W. Yan, H. Gao, Z. Yue, J. Liu, F. Wang, Finite element simulation of surface deformation of polycrystal with a rough surface under repeated load, Finite Elements in Analysis and Design 60 (2012) 64-71.

[11] A. Le Pécheur, F. Curtit, M. Clavel, J. Stephan, C. Rey, P. Bompard, Polycrystal modelling of fatigue: Pre-hardening and surface roughness effects on damage initiation for 304L stainless steel, International Journal of Fatigue 45 (2012) 48-60.

[12] G. Owolabi, R. Prasannavenkatesan, D. McDowell, Probabilistic framework for a microstructure-sensitive fatigue notch factor, International Journal of Fatigue 32 (8) (2010) 1378-1388. 
[13] L. Méric, G. Cailletaud, M. Gaspérini, F.E. calculations of copper bicrystal specimens submitted to tension-compression tests, Acta Metallurgica et Materialia 42 (3) (1994) 921-935.

[14] P. Franciosi, Etude théorique et expérimentale du comportement élastoplastique des monocristaux métalliques se déformant par glissement : modélisation pour un chargement complexe quasi statique, Ph.D. thesis, Université Paris Nord - Paris 13 (1984).

[15] C. Gérard, Mesures de champs et identification de modèles de plasticité cristalline, Ph.D. thesis, Université Paris 13 (2008).

[16] G. Bertolino, A. Constantinescu, M. Ferjani, P. Treiber, A multiscale approach of fatigue and shakedown for notched structures, Theoretical and Applied Fracture Mechanics 48 (2) (2007) $140-151$.

[17] C. Geuzaine, J. Remacle, Gmsh: A 3-d finite element mesh generator with built-in pre-and post-processing facilities, International Journal for Numerical Methods in Engineering 79 (11) (2009) 1309-1331.

[18] O. Diard, S. Leclercq, G. Rousselier, G. Cailletaud, Evaluation of finite element based analysis of 3D multicrystalline aggregates plasticity: Application to crystal plasticity model identification and the study of stress and strain fields near grain boundaries, International Journal of Plasticity 21 (4) (2005) 691-722.

[19] J. Besson, R. Leriche, R. Foerch, G. Cailletaud, Object-oriented programming applied to the finite element method part II. application to material behaviors, Revue Européenne des Éléments 7 (5) (1998) 567-588.

[20] K. Dang-Van, Macro-micro approach in high-cycle multiaxial fatigue, in: ASTM Special Technical Publication, 1993, pp. 120-130.

[21] I. Papadopoulos, A new criterion of fatigue strength for out-of-phase bending and torsion of hard metals, International Journal of Fatigue 16 (6) (1994) 377-384.

[22] F. Morel, N. Huyen, Plasticity and damage heterogeneity in fatigue, Theoretical and Applied Fracture Mechanics 49 (1) (2008) 98-127. 
[23] A. Bernasconi, I. Papadopoulos, Efficiency of algorithms for shear stress amplitude calculation in critical plane class fatigue criteria, Computational Materials Science 34 (4) (2005) 355-368.

[24] A. M. Freudenthal, E. J. Gumbel, On the statistical interpretation of fatigue tests, Proceedings of the Royal Society of London. Series A. Mathematical and Physical Sciences 216 (1126) (1953) 309-332.

[25] Y. Li, V. Aubin, C. Rey, P. Bompard, Polycrystalline numerical simulation of variable amplitude loading effects on cyclic plasticity and microcrack initiation in austenitic steel 304l, International Journal of Fatigue 42 (0) (2012) 71 - 81.

[26] C. P. Przybyla, D. L. McDowell, Simulated microstructure-sensitive extreme value probabilities for high cycle fatigue of duplex ti6al4v, International Journal of Plasticity $27(12)(2011) 1871-1895$.

[27] M. Endo, Y. Murakami, Effects of an artificial small defect on torsional fatigue strength of steels, Journal of Engineering Materials and Technology 109 (2) (1987) 124-129.

[28] T. Billaudeau, Y. Nadot, G. Bezine, Multiaxial fatigue limit for defective materials: mechanisms and experiments, Acta Materialia 52 (13) (2004) 3911 - 3920.

[29] M. Endo, The multiaxial fatigue strength of specimens containing small defects, in: M. d. F. Andrea Carpinteri, A. Spagnoli (Eds.), European Structural Integrity Society, Vol. Volume 31 of Biaxial/Multiaxial Fatigue and Fracture 6th International Conference on Biaxial/Multiaxial Fatigue and Fracture, Elsevier, 2003, pp. 243-264. 


\section{List of Figures}

1 Representation of some mechanical quantities and vectors in (a) a FCC unit cell and (b) a slip plane. . . . . . . . . . . . . . . . . . . 28

2 Specimen geometry and modelled zone. . . . . . . . . . . . . . . 28

3 Mesh of a 2-dimensional notched microstructure of approximately 200 grains embedded in an isotropic matrix. . . . . . . . . . . . . . . . . . . . 29

4 Geometry of the holed microstructure. . . . . . . . . . . . . . . . . . . 29

5 Accumulated equivalent plastic slip field at the end of the $10^{\text {th }}$ loading cycle for the same microstructure, loaded at the macroscopic fatigue limit level, with different notch radii and orientation sets. . . . . . . . . . . . .

6 Mechanical responses, in terms of $\tau_{a^{-}} \sigma_{n, a}$, of each slip planes obtained from the FE simulations of notched microstructures loaded in fully reversed tension at the fatigue limit level. . . . . . . . . . . . . . . . . . . . . . .

$7 \quad$ Mechanical responses, in terms of $\sigma_{n, m^{-}} \sigma_{n, a}$, of each slip planes obtained from the FE simulations of notched microstructures, using the crystal plasticity model, loaded in fully reversed tension at the fatigue limit level. . . . . . . . . .

8 Stereographic projection, in the plane of normal $\underline{e}_{3}$, of the unit vector normal to the most critical slip plane of each grain and associated failure probability $P_{F g}$ obtained with notched microstructures loaded in fully reversed tension. .

9 Experimental fatigue limits in fully reversed tension for different notch radii [1] and predictions of the fatigue criteria, for the notched microstructures, obtained with (a) the isotropic elasticity, (b) the cubic elasticity and (c) the crystal plasticity with the cubic elasticity. . . . . . . . . . . .

10 Mechanical responses, in terms of $\tau_{a^{-}} \sigma_{n, a}$, of each slip planes obtained from the FE simulations of holed microstructures in fully reversed tension at the fatigue limit level. . . . . . . . . . . . . . . . . .

11 Mechanical responses, in terms of $\tau_{a}-\sigma_{n, a}$, of each slip planes obtained from the FE simulations of holed microstructures in fully reversed shear at the fatigue limit level. . . . . . . . . . . . . . . . . . . . . . . . . . . . 36 
12 Stereographic projection, in the plane of normal $\underline{e}_{3}$, of the unit vector normal to the most critical slip plane of each grain and associated failure probability $P_{F g}$ obtained with holed microstructures loaded in fully reversed tension. . . 37

13 Stereographic projection, in the plane of normal $\underline{e}_{3}$, of the unit vector normal to the most critical slip plane of each grain and associated failure probability $P_{F g}$ obtained with holed microstructures loaded in fully reversed shear. . . . 38

14 Predictions of the probabilistic fatigue criterion, for the holed microstructures loaded in fully reversed tension and in fully reversed shear for each elastic constitutive models. . . . . . . . . . . . . . . . .

15 Evolution of the ratio $\phi$ predicted by the probabilistic fatigue criterion with

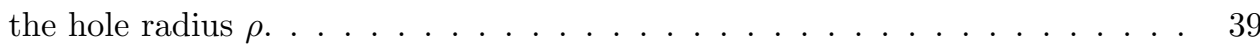




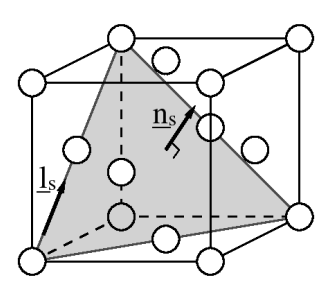

(a)

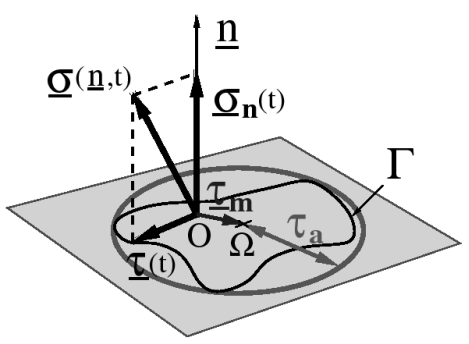

(b)

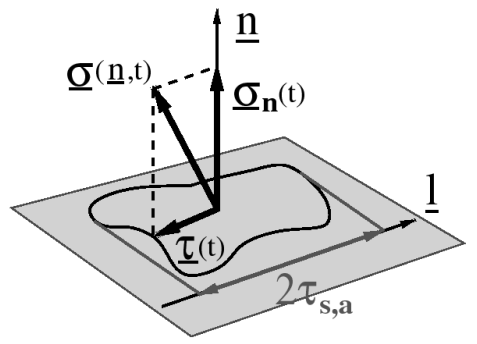

Figure 1: Representation of some mechanical quantities and vectors in (a) a FCC unit cell and (b) a slip plane.

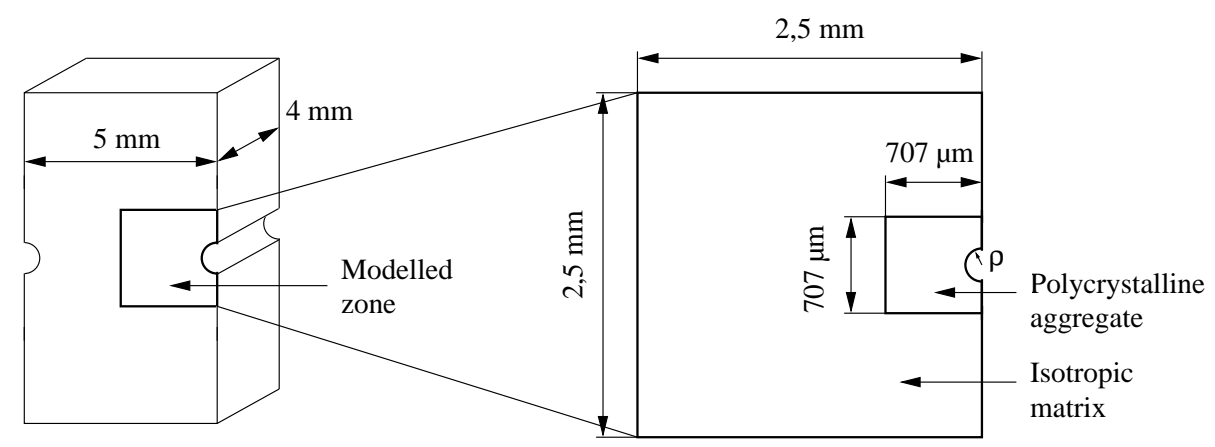

Figure 2: Specimen geometry and modelled zone. 


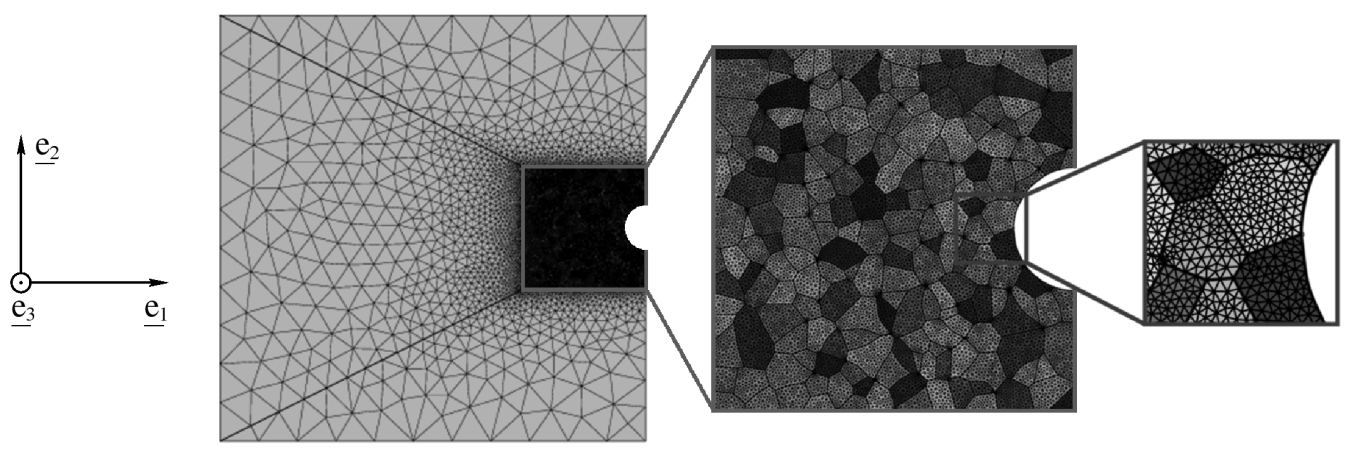

Figure 3: Mesh of a 2-dimensional notched microstructure of approximately 200 grains embedded in an isotropic matrix.

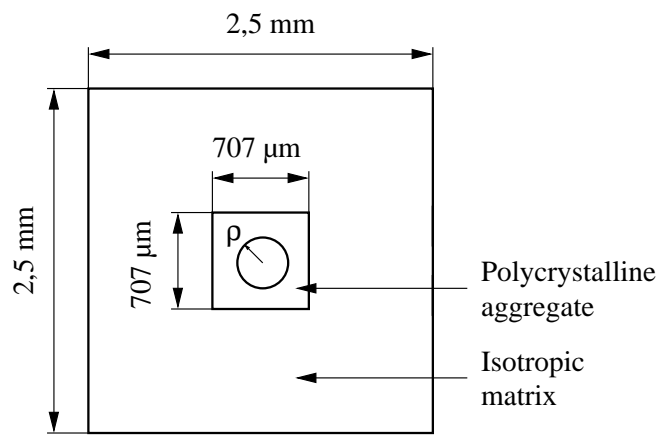

Figure 4: Geometry of the holed microstructure. 

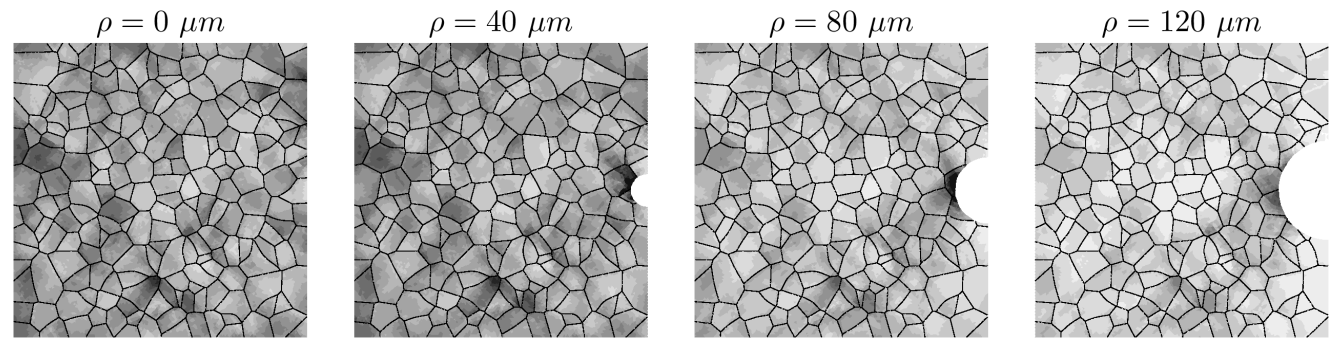

(a) Orientation set $n^{\circ} 1$
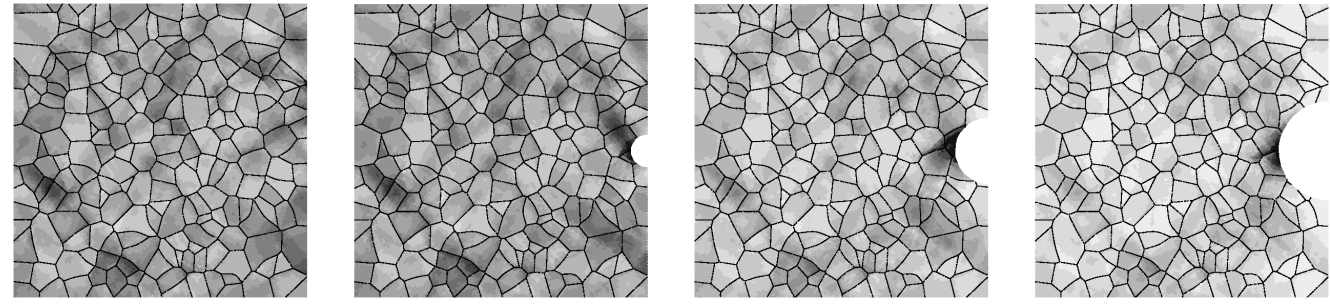

(b) Orientation set $n^{\circ} 2$
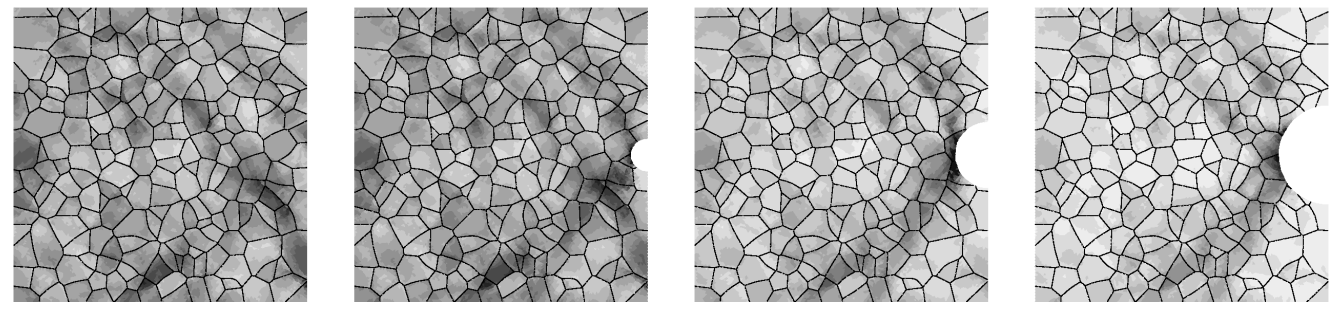

(c) Orientation set $n^{\circ} 3$

Cumulated equivalent plastic slip

$\begin{array}{llllllllllllll}0 & 0.05 & 0.1 & 0.15 & 0.2 & 0.25 & 0.3 & 0.35 & 0.4 & 0.45 & 0.5 & 0.55 & 0.6 & 0.65\end{array}$

Figure 5: Accumulated equivalent plastic slip field at the end of the $10^{\text {th }}$ loading cycle for the same microstructure, loaded at the macroscopic fatigue limit level, with different notch radii and orientation sets. 
Isotropic elasticity
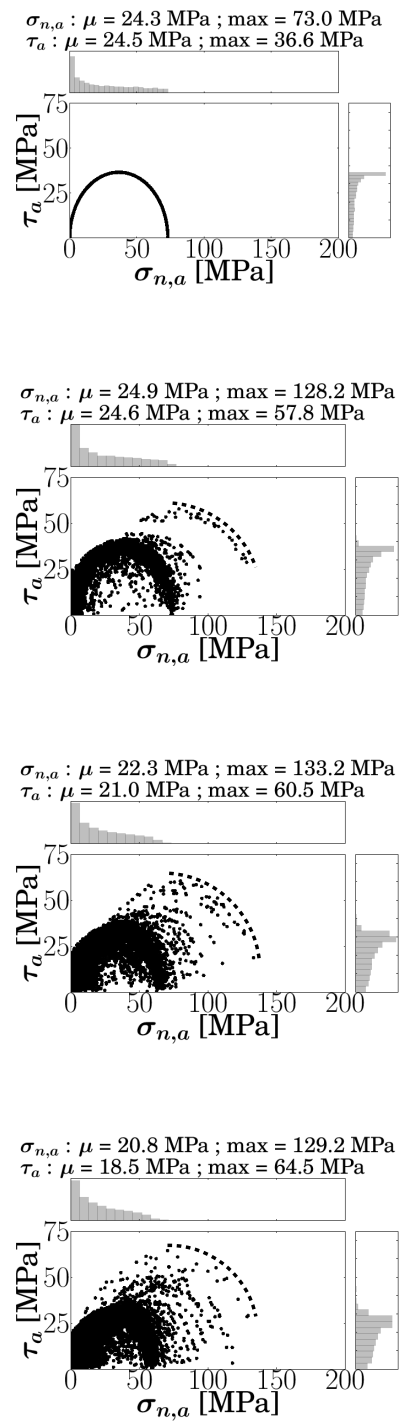

Cubic elasticity

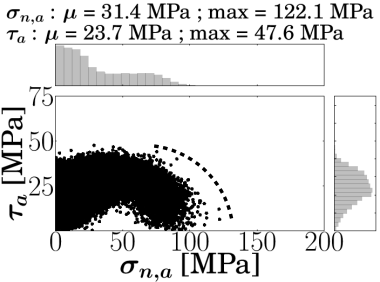

(a) $\rho=0 \mu m$

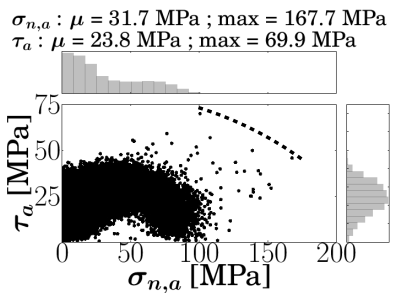

(b) $\rho=40 \mu \mathrm{m}$

$\sigma_{n, a}: \mu=27.6 \mathrm{MPa} ; \max =163.7 \mathrm{MPa}$ $\tau_{a}: \mu=20.4 \mathrm{MPa} ; \max =67.0 \mathrm{MPa}$

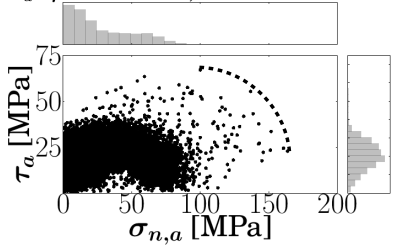

(c) $\rho=80 \mu m$

$\sigma_{n, a}: \mu=25.0 \mathrm{MPa} ; \max =187.5 \mathrm{MPa}$

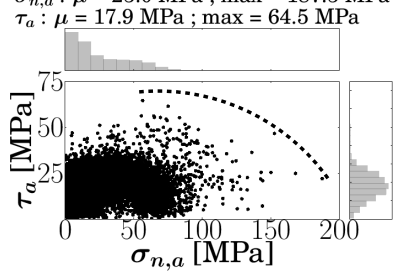

(d) $\rho=120 \mu m$
Cubic elasticity and crystal plasticity
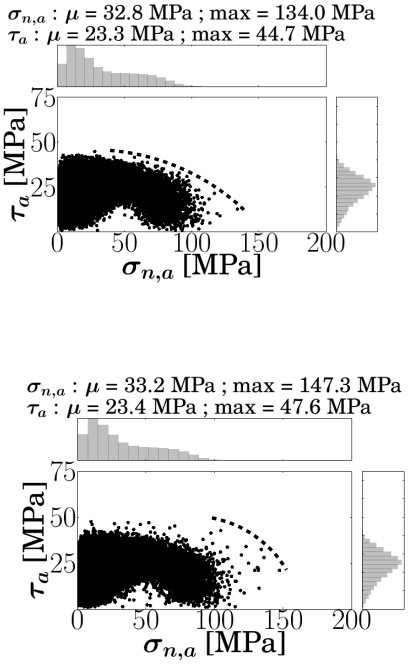

$\sigma_{n, a}: \mu=28.7 \mathrm{MPa} ; \max =139.9 \mathrm{MPa}$ $\tau_{a}: \mu=19.8 \mathrm{MPa} ; \max =45.4 \mathrm{MPa}$

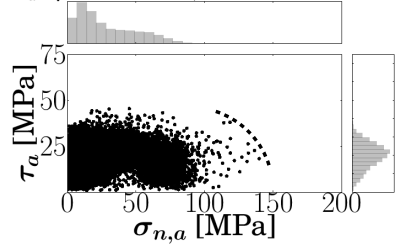

$\sigma_{n, a}: \mu=26.0 \mathrm{MPa} ; \max =193.8 \mathrm{MPa}$ $\tau_{a}: \mu=17.4 \mathrm{MPa} ; \max =62.0 \mathrm{MPa}$

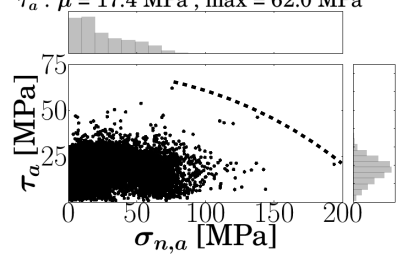

Figure 6: Mechanical responses, in terms of $\tau_{a^{-}} \sigma_{n, a}$, of each slip planes obtained from the FE simulations of notched microstructures loaded in fully reversed tension at the fatigue limit level. 


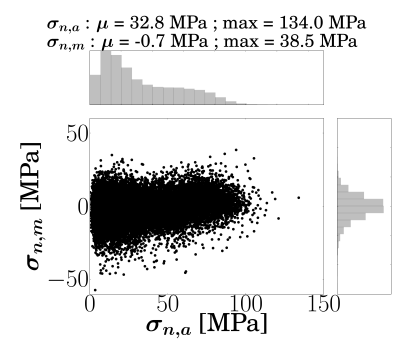

(a) $\rho=0 \mu m$

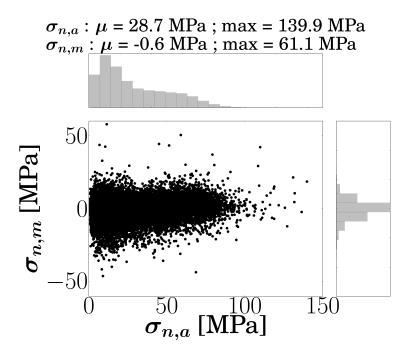

(c) $\rho=80 \mu m$

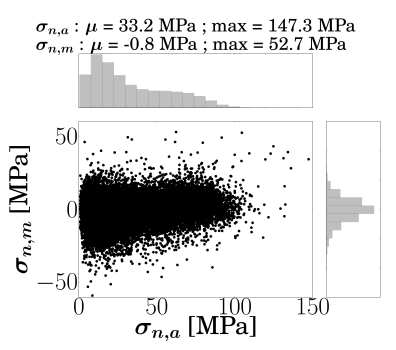

(b) $\rho=40 \mu \mathrm{m}$

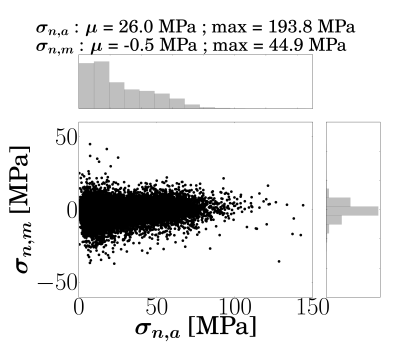

(d) $\rho=120 \mu \mathrm{m}$

Figure 7: Mechanical responses, in terms of $\sigma_{n, m}-\sigma_{n, a}$, of each slip planes obtained from the FE simulations of notched microstructures, using the crystal plasticity model, loaded in fully reversed tension at the fatigue limit level. 

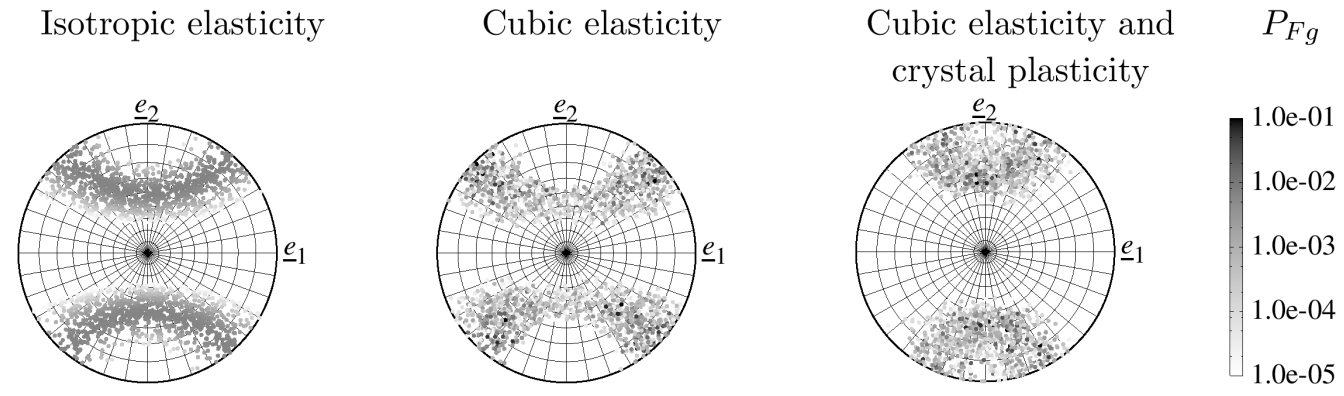

(a) $\rho=0 \mu m$
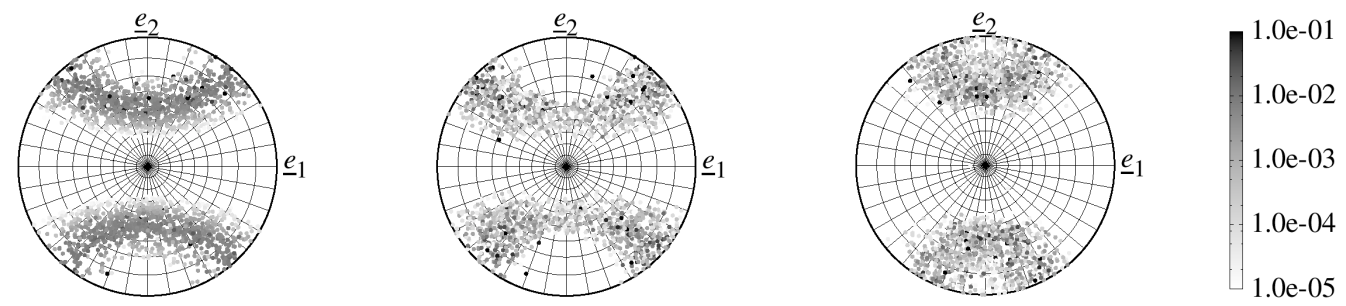

(b) $\rho=40 \mu m$
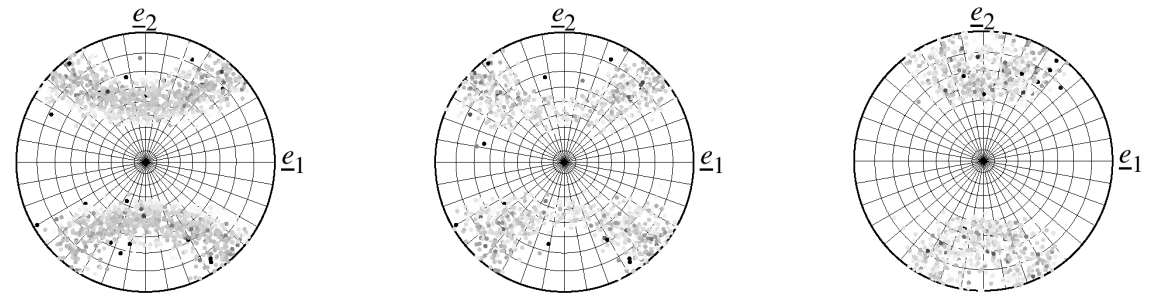

$1.0 \mathrm{e}-01$
$1.0 \mathrm{e}-02$
$1.0 \mathrm{e}-03$
$1.0 \mathrm{e}-04$
$1.0 \mathrm{e}-05$

(c) $\rho=80 \mu m$
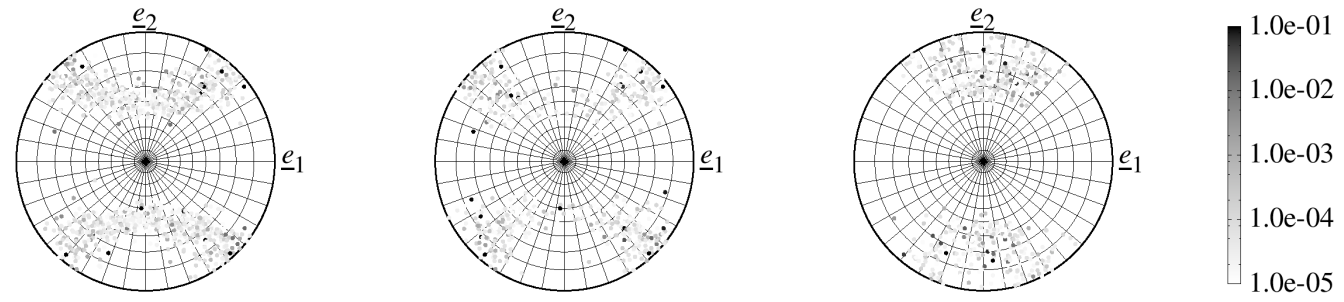

(d) $\rho=120 \mu m$

Figure 8: Stereographic projection, in the plane of normal $\underline{e}_{3}$, of the unit vector normal to the most critical slip plane of each grain and associated failure probability $P_{F g}$ obtained with notched microstructures loaded in fully reversed tension. 
Critical plane criterion Integral criterion

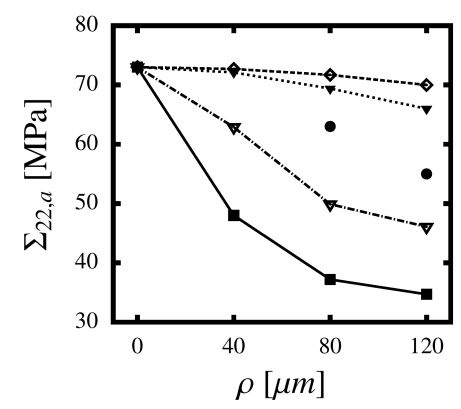

(a) Isotropic elasticity
Prob. criterion $(\mathrm{m}=5) \quad \cdots \cdot \boldsymbol{\nabla} \cdot \cdots \cdot$ Prob. criterion $(m=20)$

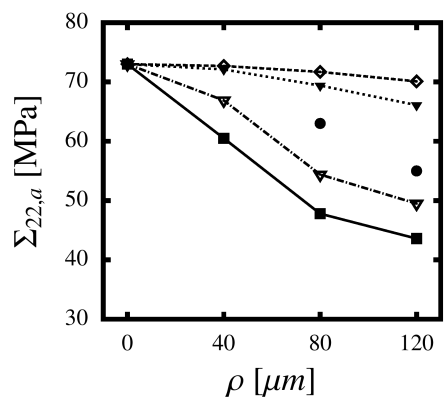

(b) Cubic elasticity
Experimental data

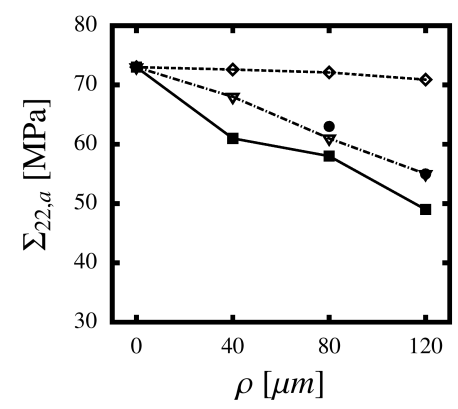

(c) Cubic elasticity and crystal plasticity

Figure 9: Experimental fatigue limits in fully reversed tension for different notch radii [1] and predictions of the fatigue criteria, for the notched microstructures, obtained with (a) the isotropic elasticity, (b) the cubic elasticity and (c) the crystal plasticity with the cubic elasticity. 

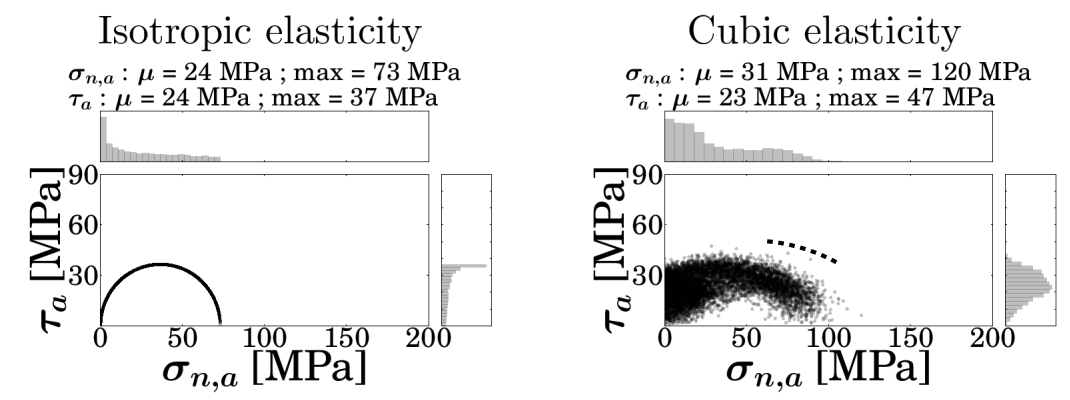

(a) $\rho=0 \mu m$
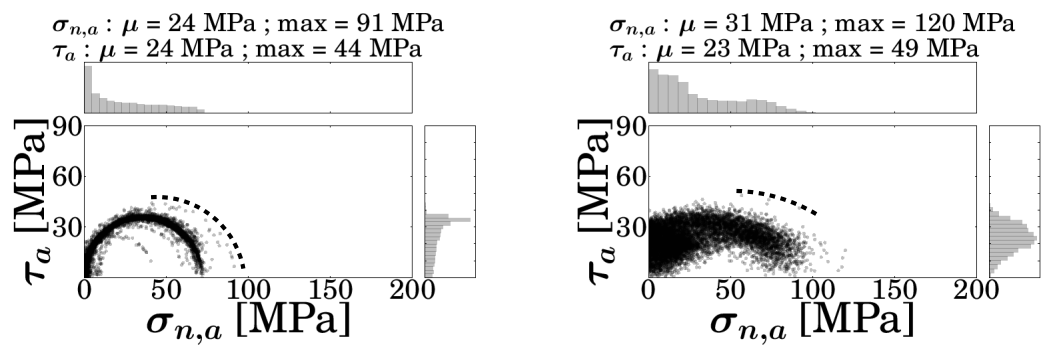

(b) $\rho=28.3 \mu \mathrm{m}$
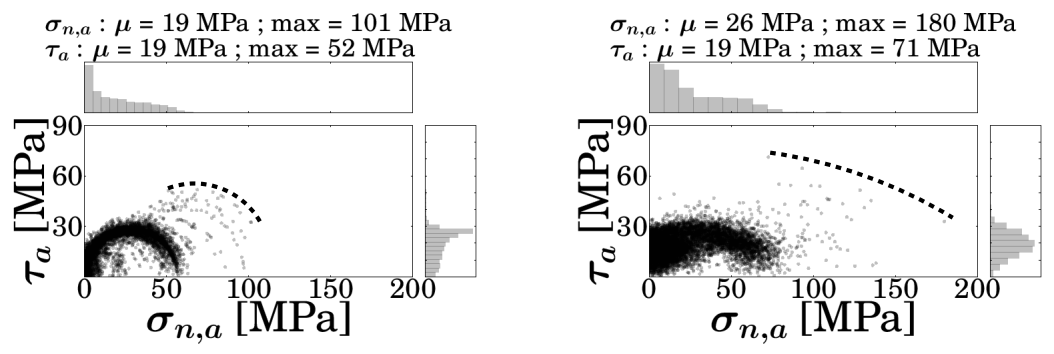

(c) $\rho=56.6 \mu m$
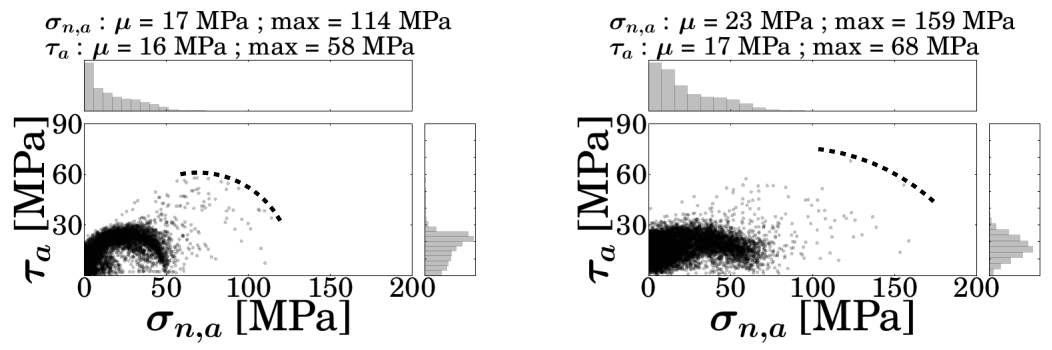

(d) $\rho=84.9 \mu \mathrm{m}$

Figure 10: Mechanical responses, in terms of $\tau_{a}-\sigma_{n, a}$, of each slip planes obtained from the FE simulations of holed microstructures in fully reversed tension at the fatigue limit level. 

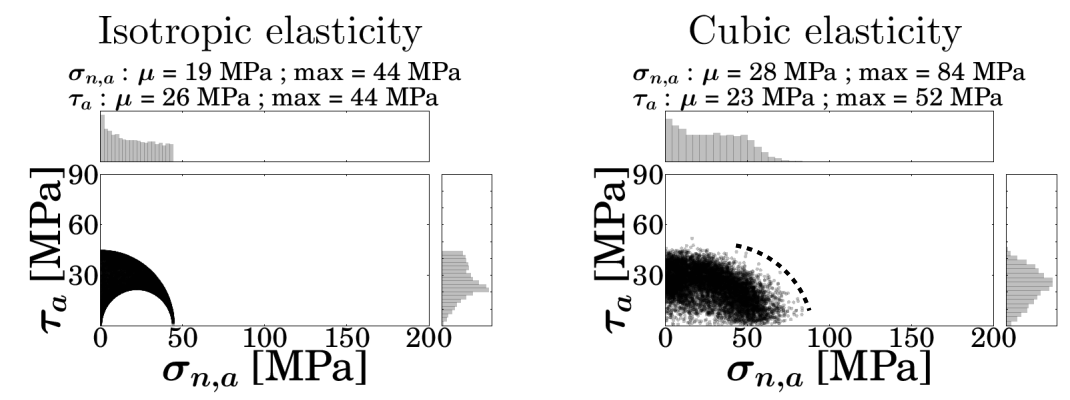

(a) $\rho=0 \mu m$
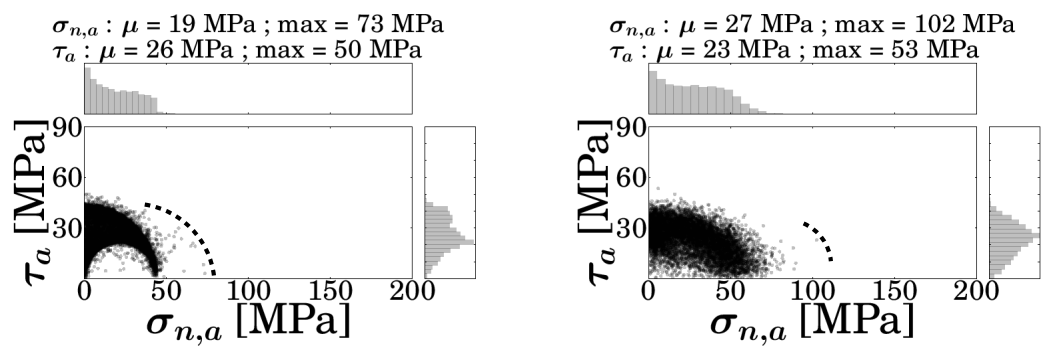

(b) $\rho=28.3 \mu \mathrm{m}$
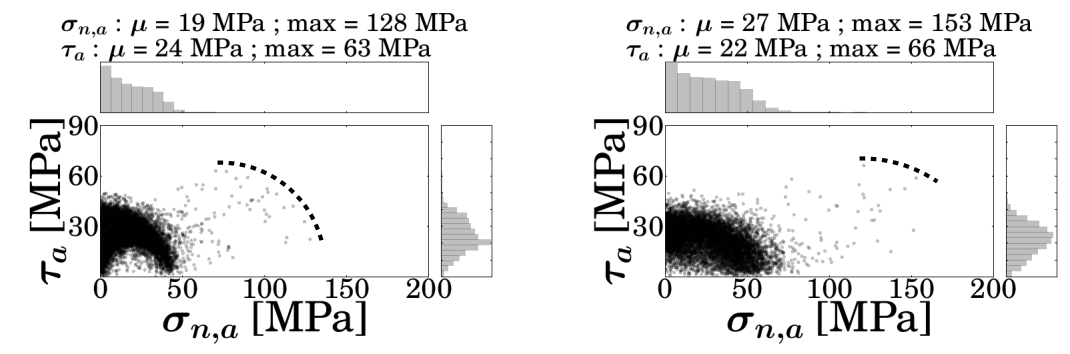

(c) $\rho=56.6 \mu \mathrm{m}$
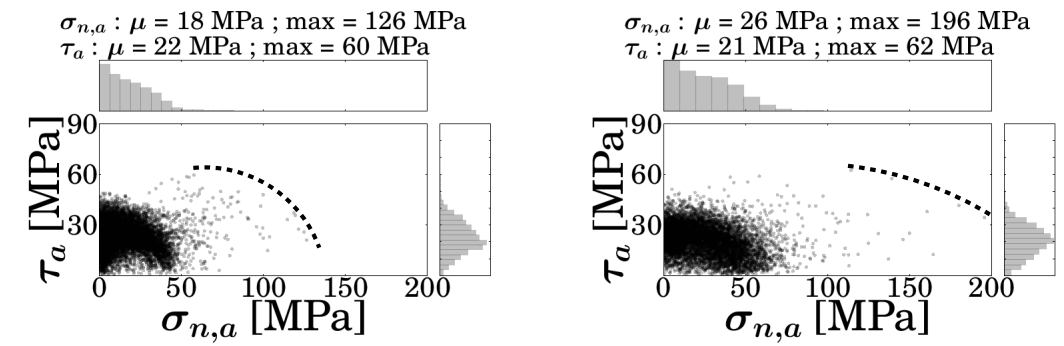

(d) $\rho=84.9 \mu \mathrm{m}$

Figure 11: Mechanical responses, in terms of $\tau_{a}-\sigma_{n, a}$, of each slip planes obtained from the FE simulations of holed microstructures in fully reversed shear at the fatigue limit level. 


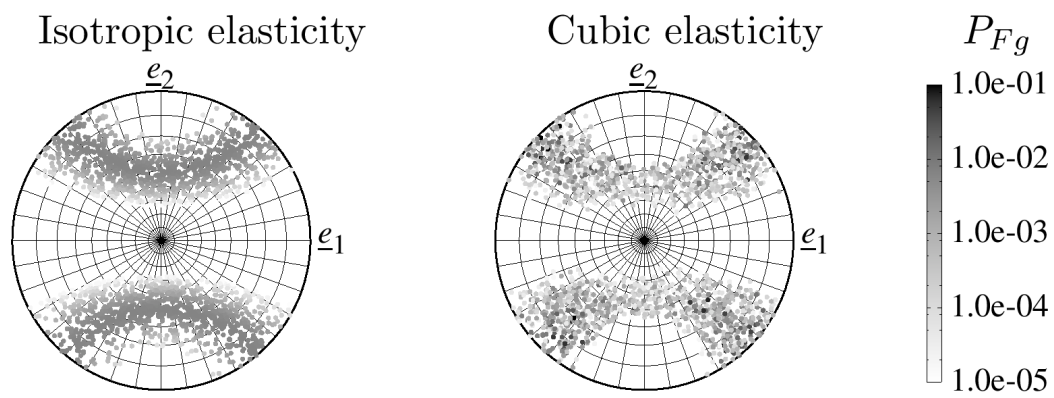

(a) $\rho=0 \mu m$
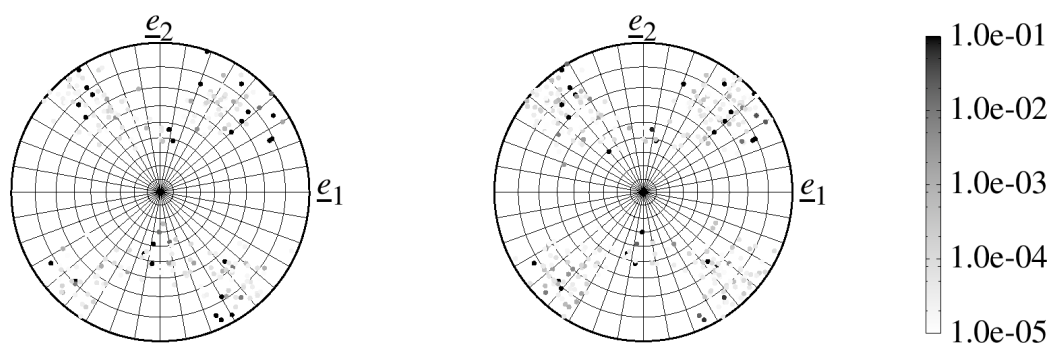

(b) $\rho=84.9 \mu m$

Figure 12: Stereographic projection, in the plane of normal $\underline{e}_{3}$, of the unit vector normal to the most critical slip plane of each grain and associated failure probability $P_{F g}$ obtained with holed microstructures loaded in fully reversed tension. 

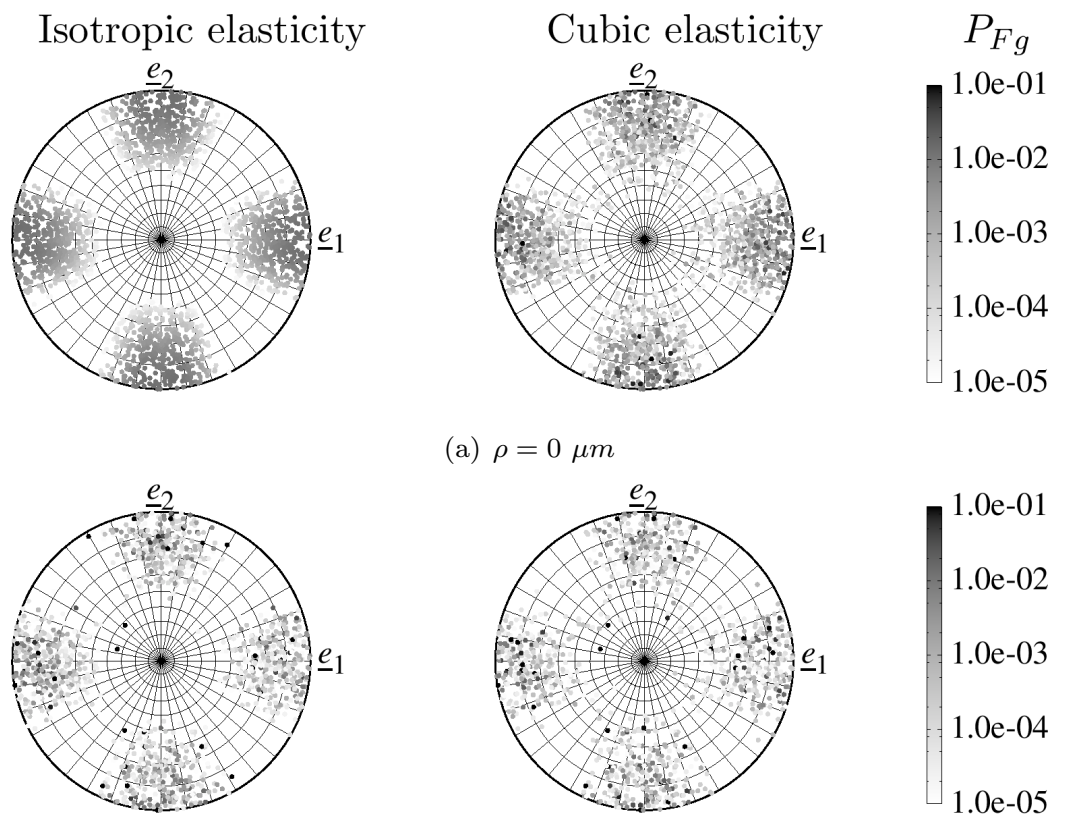

(a) $\rho=0 \mu m$

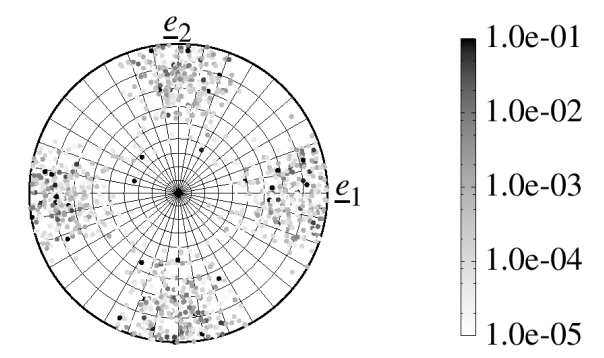

(b) $\rho=84.9 \mu \mathrm{m}$

Figure 13: Stereographic projection, in the plane of normal $\underline{e}_{3}$, of the unit vector normal to the most critical slip plane of each grain and associated failure probability $P_{F g}$ obtained with holed microstructures loaded in fully reversed shear. 


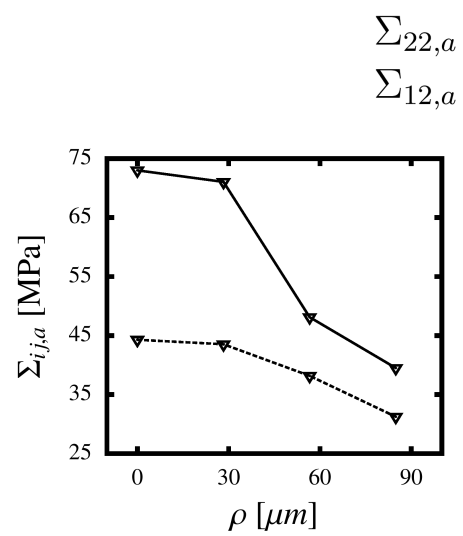

(a) Isotropic elasticity

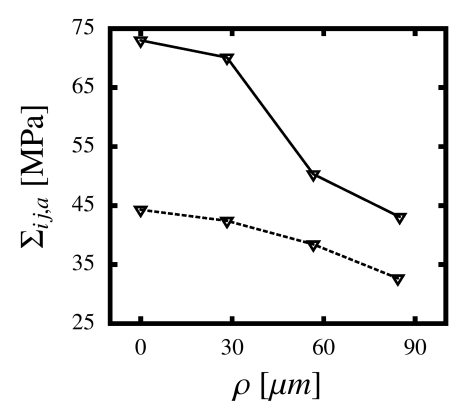

(b) Cubic elasticity

Figure 14: Predictions of the probabilistic fatigue criterion, for the holed microstructures loaded in fully reversed tension and in fully reversed shear for each elastic constitutive models.

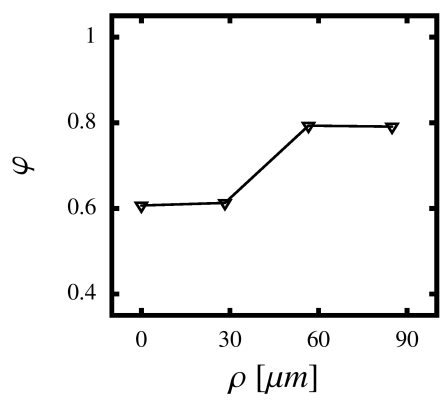

(a) Isotropic elasticity

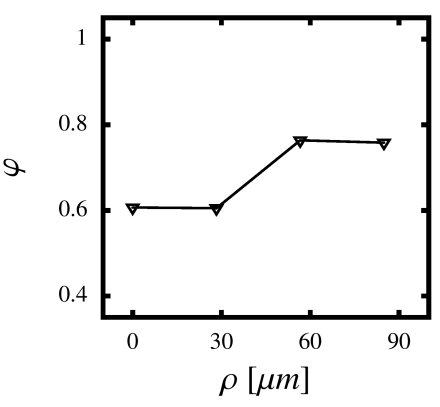

(b) Cubic elasticity

Figure 15: Evolution of the ratio $\phi$ predicted by the probabilistic fatigue criterion with the hole radius $\rho$. 


\section{List of Tables}

1 Material parameters used for the annealed electrolytic copper. . . . . . . . . . 41

2 Material parameters used for the isotropic matrix. . . . . . . . . . . . . . 41 


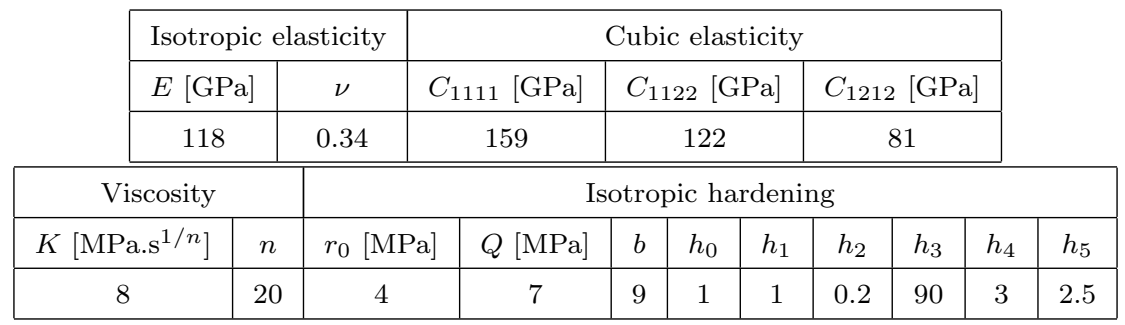

Table 1: Material parameters used for the annealed electrolytic copper.

\begin{tabular}{|c|c|c|c|c|}
\hline$\Sigma_{y}[\mathrm{MPa}]$ & $Q[\mathrm{MPa}]$ & $b$ & $C[\mathrm{MPa}]$ & $\gamma$ \\
\hline 26.3 & 100.0 & 5.36 & 1316 & 168.4 \\
\hline
\end{tabular}

Table 2: Material parameters used for the isotropic matrix. 